Supporting Information for

\title{
Interpreting the Paramagnetic NMR Spectra of Potential Ru(III) Metallodrugs: Synergy between Experiment and Relativistic DFT Calculations
}

Jan Novotný, ${ }^{\dagger}$ Martin Sojka, ${ }^{\dagger, t}$ Stanislav Komorovsky, ${ }^{\$}$ Marek Nečas, ${ }^{\dagger, t}$ and Radek Marek ${ }^{\dagger, \ldots, *}$

${ }^{+}$CEITEC - Central European Institute of Technology, Masaryk University, Kamenice 5, CZ62500 Brno, Czech Republic

$¥$ Department of Chemistry, Faculty of Science, Masaryk University, Kamenice 5, CZ - 62500

Brno, Czech Republic

${ }^{\$}$ Centre for Theoretical and Computational Chemistry, Department of Chemistry, UiT - The Arctic University of Norway, N-9037 Troms $\phi$, Norway 


\section{METHODS}
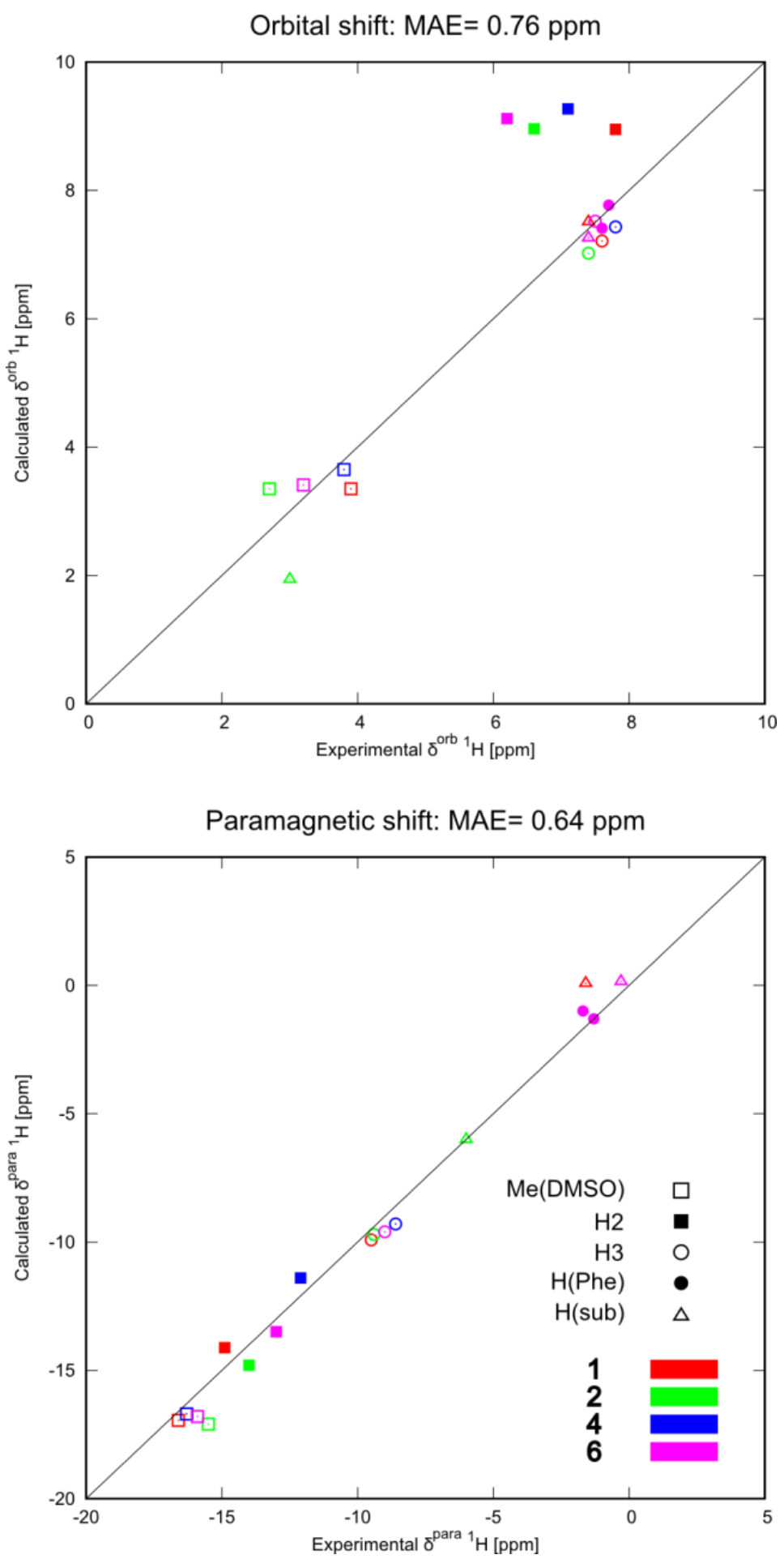

Figure S1a. Correlation between the experimental and DFT-calculated ${ }^{1} \mathrm{H}$ NMR chemical shifts $\delta^{\text {orb }}$ (top) and $\delta^{\text {para }}$ (bottom) for compounds 1, 2, 4, and 6. MAE - Mean Absolute Error. 


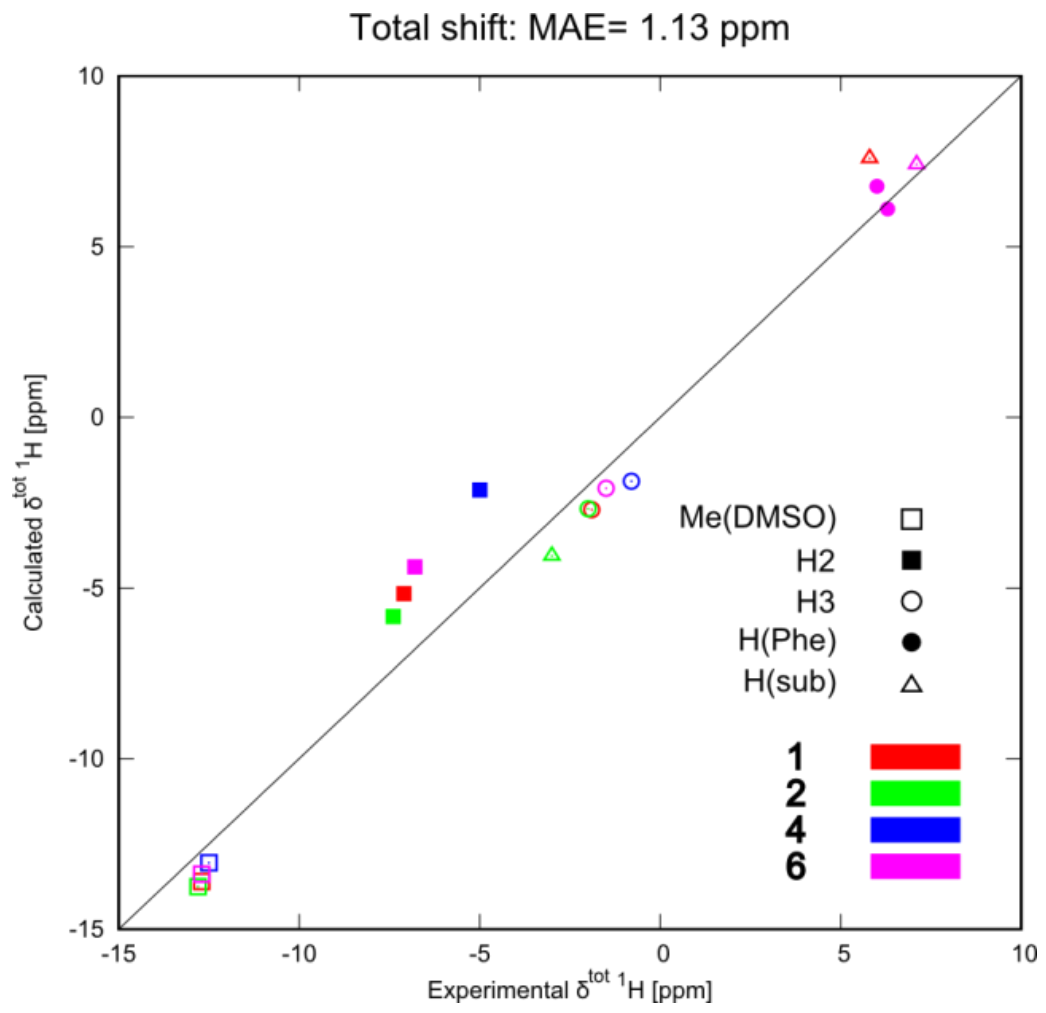

Figure S1b. Correlation between the experimental and DFT-calculated total ${ }^{1} \mathrm{H}$ NMR chemical shifts $\delta^{\text {tot }}$ for compounds 1, 2, 4, and 6. MAE - Mean Absolute Error.

\section{Comment on Figure 1}

The largest deviation of the calculated ${ }^{1} \mathrm{H}$ NMR chemical shifts from the experimental values is observed for $\mathrm{H} 2$ atoms close to the paramagnetic ruthenium center (full squares in Figure $\mathrm{S} 1 \mathrm{~b}$ ). This deviation is traced to the orbital contribution in Figure S1a (full squares in the top plot) and can be related to the SO-ZORA calculation of the orbital shift $\left(\delta^{\text {orb }}\right)$ on an artificial closed-shell system, missing kernel, and/or an insufficient description of the solvent effects (see Figure S3). 


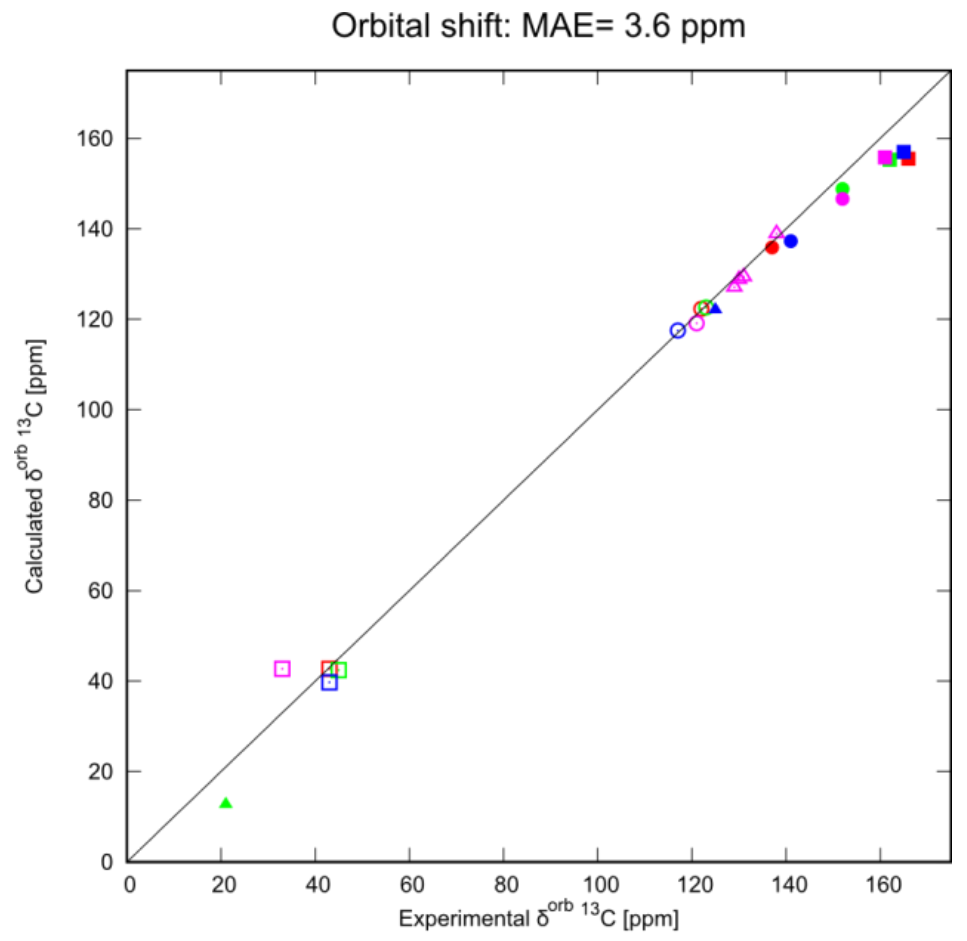

Paramagnetic shift: $\mathrm{MAE}=4.0 \mathrm{ppm}$

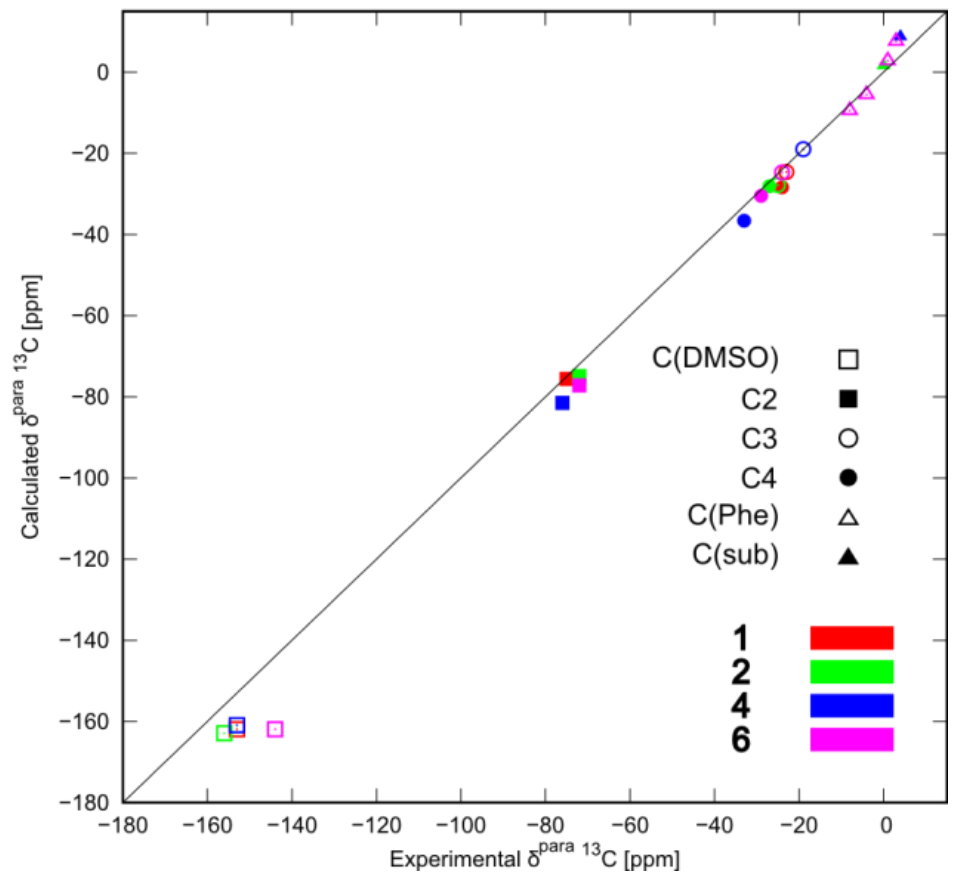

Figure S2a. Correlation between the experimental and DFT-calculated ${ }^{13} \mathrm{C}$ NMR chemical shifts $\delta^{\text {orb }}$ (top) and $\delta^{\text {para }}$ (bottom) for compounds 1, 2, 4, and 6. MAE - Mean Absolute Error. 


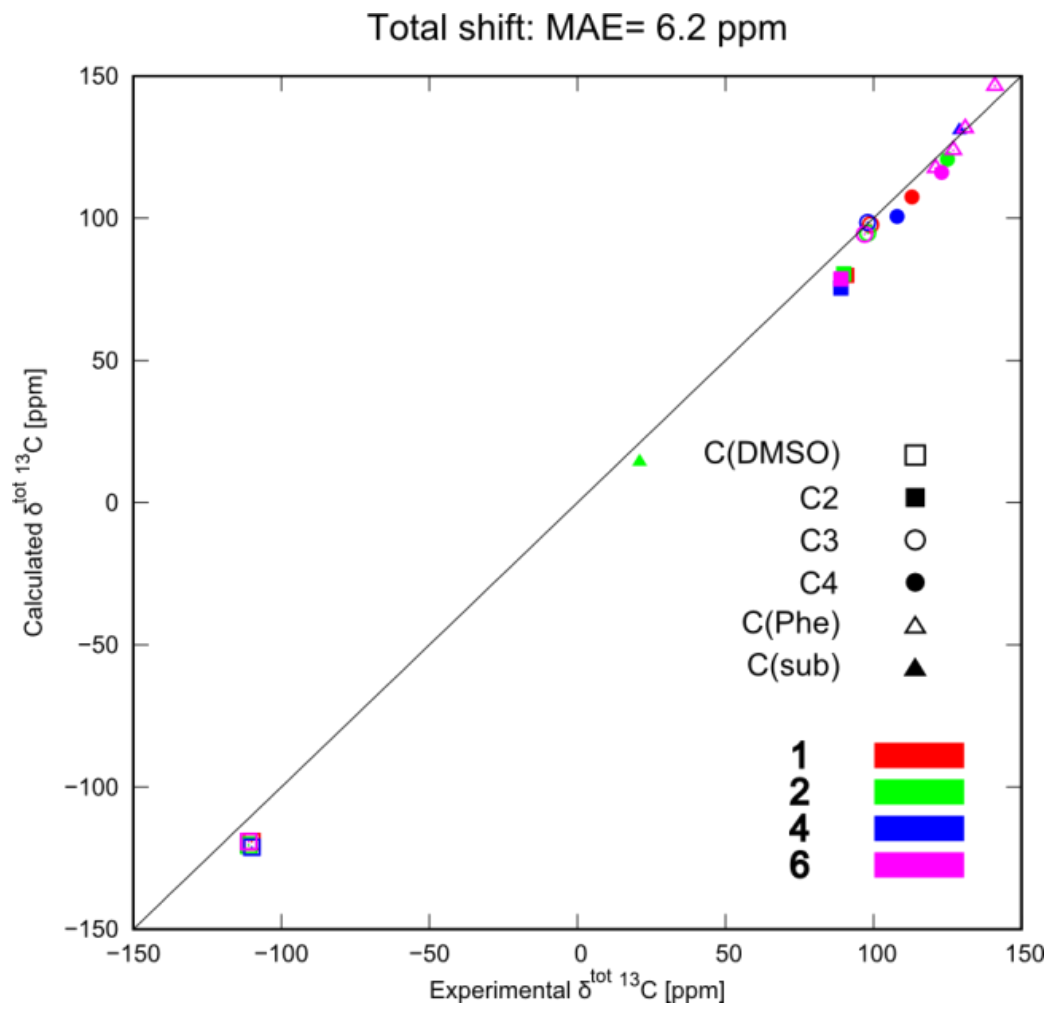

Figure S2b. Correlation between the experimental and DFT-calculated total ${ }^{13} \mathrm{C}$ NMR chemical shifts $\delta^{\text {tot }}$ for compounds 1, 2, 4, and 6. MAE - Mean Absolute Error. 

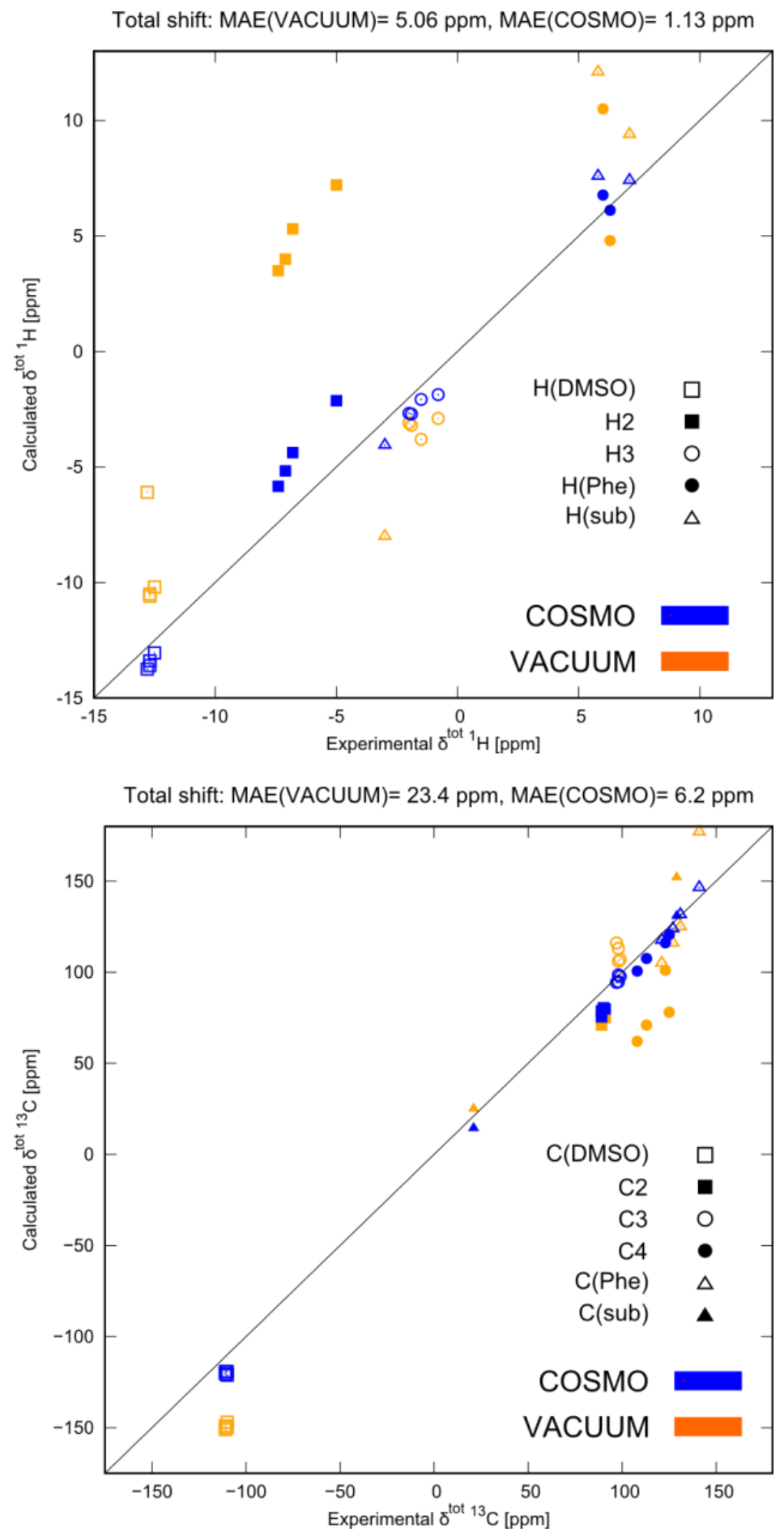

Figure S3. Correlation between the experimental and DFT-calculated total NMR chemical shifts $\left(\delta^{\text {tot }}\right)$. The ${ }^{1} \mathrm{H}$ (top) and ${ }^{13} \mathrm{C}$ (bottom) NMR chemical shifts for compounds 1, 2, 4, and 6 were calculated in vacuum or by using the COSMO model of solvent (DMF). MAE - Mean Absolute Error. 

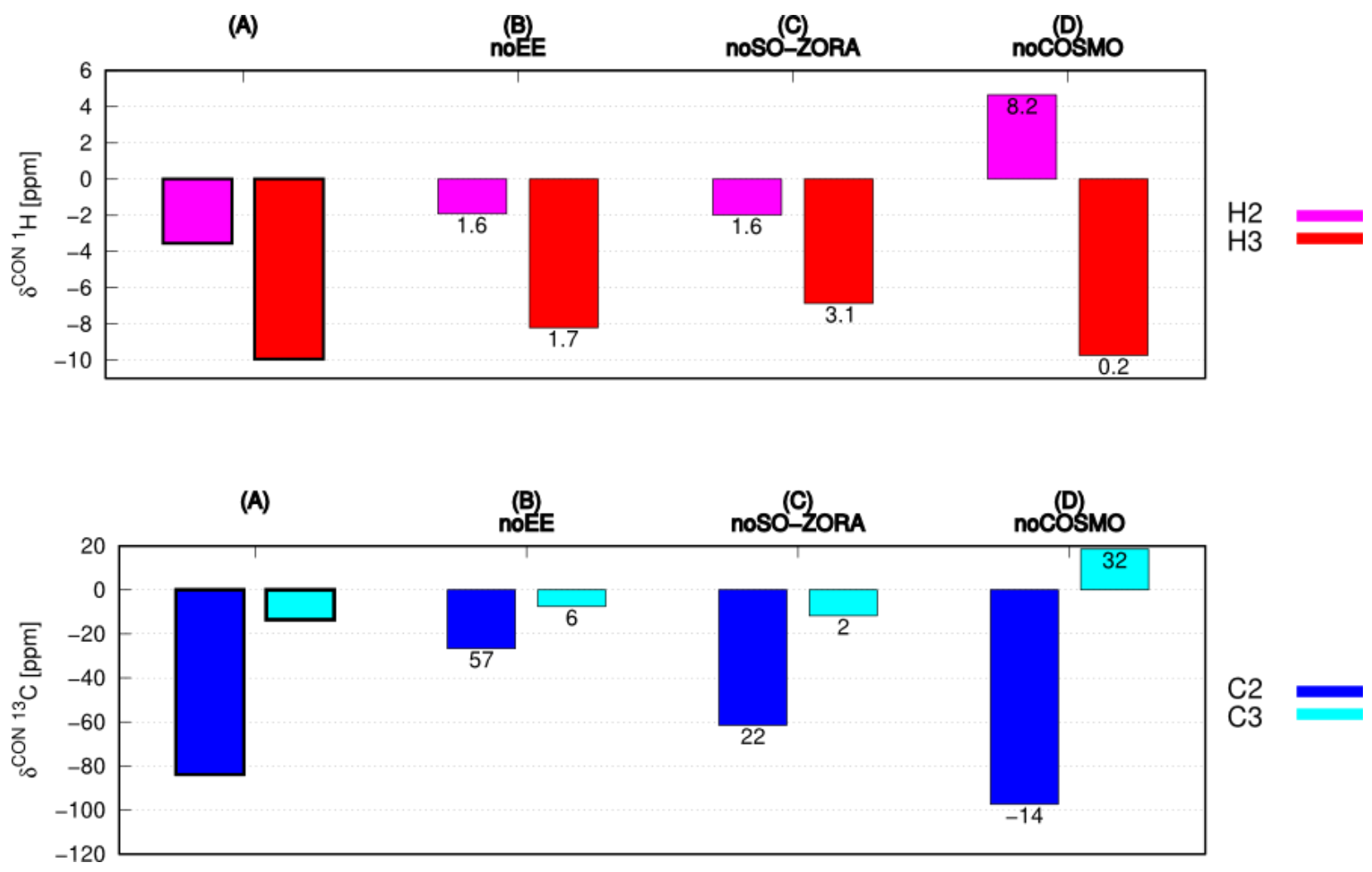

Figure S4. The effects of various factors on the contact contribution $\left(\delta^{\mathrm{con}}\right)$ to the paramagnetic ${ }^{1} \mathrm{H}$ (top, $\mathrm{H}-2$ and $\mathrm{H}-3$ ) and ${ }^{13} \mathrm{C}$ (bottom, C-2 and C-3) NMR chemical shifts in complex 3.
(A): PBE0/SO-ZORA/TZ2P/COSMO level (used to produce the calculations in the main article).
(B): PBE (0 \% of exact-exchange admixture); numbers indicate the differences from (A).
(C): Nonrelativistic (without SO-ZORA); numbers indicate the differences from (A).
(D): in vacuum (without COSMO); numbers indicate the differences from (A). 


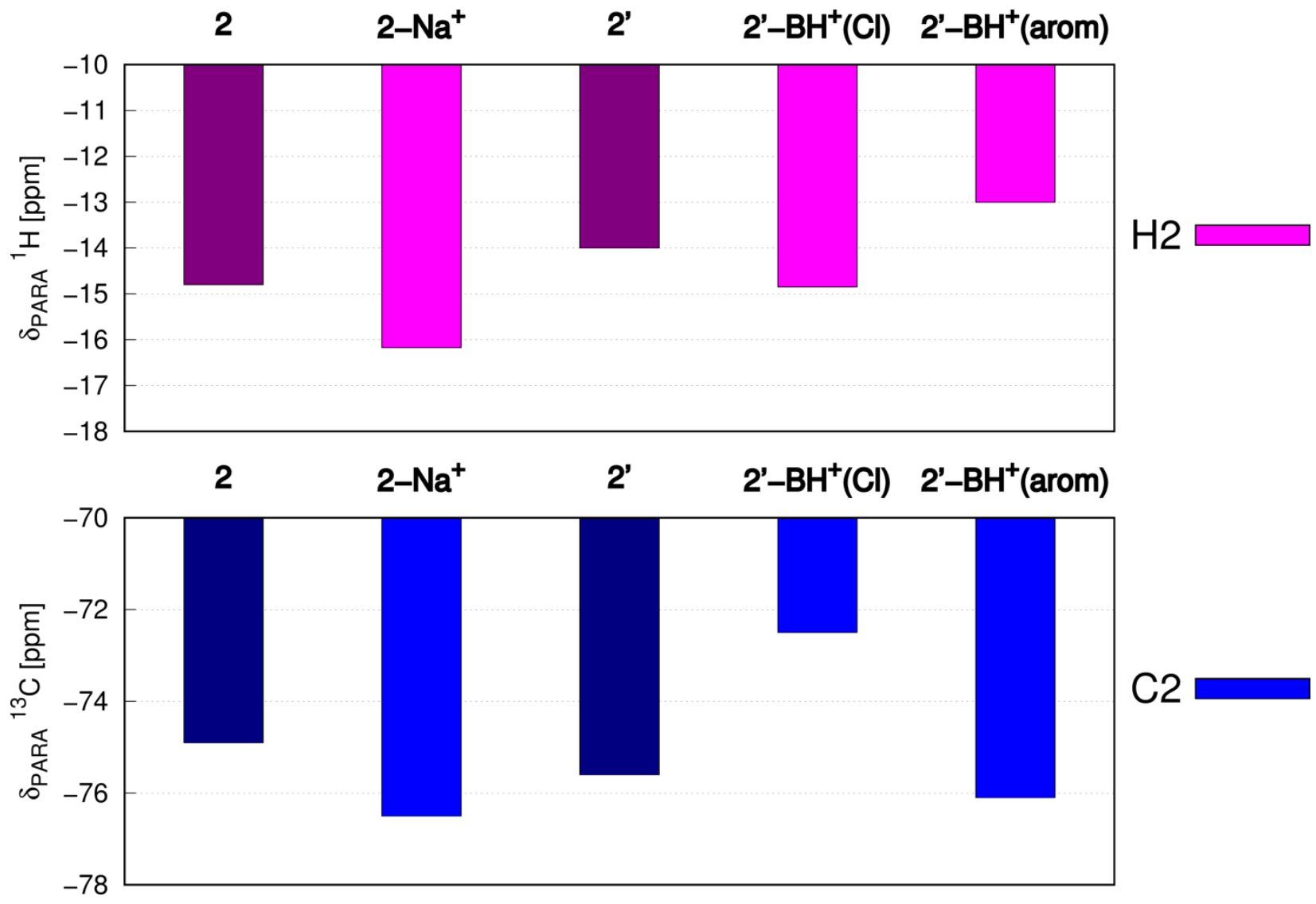

Figure S5. The effect of the presence and nature of a counter-cation on the paramagnetic NMR chemical shift of $\mathrm{H} 2$ and $\mathrm{C} 2$ atoms in complex 2.

Label 2: The DFT-optimized anion of the ruthenium complex 2 (used to produce the calculations in the main article), 2-Na': the DFT-optimized complex 2 with $\mathrm{Na}^{+}$.

Label 2': X-ray crystal structure of the anion of the ruthenium complex 2 with optimized hydrogen atoms (for Cartesian coordinates, see below), $\mathbf{2}^{\prime}-\mathbf{B H}^{+}(\mathbf{C l})$ : X-ray structure of the ion pair containing $\mathrm{MePyH}^{+}$in the vicinity of $\mathrm{Cl}$ ligands (for Cartesian coordinates, see below), 2'$\mathbf{B H}^{+}(\mathbf{a r o m})$ : X-ray structure of the ion pair containing $\mathrm{MePyH}^{+}$stacked with the MePy ligand of the anion (for Cartesian coordinates, see below). Positions of all $\mathrm{H}$ atoms were DFT-optimized in the 2' structures derived from the crystallographic data. 


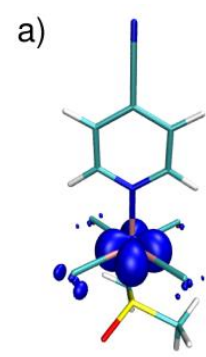

0.006

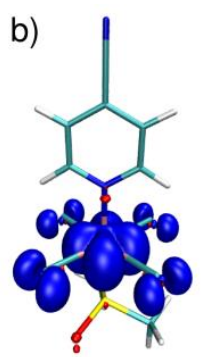

0.002
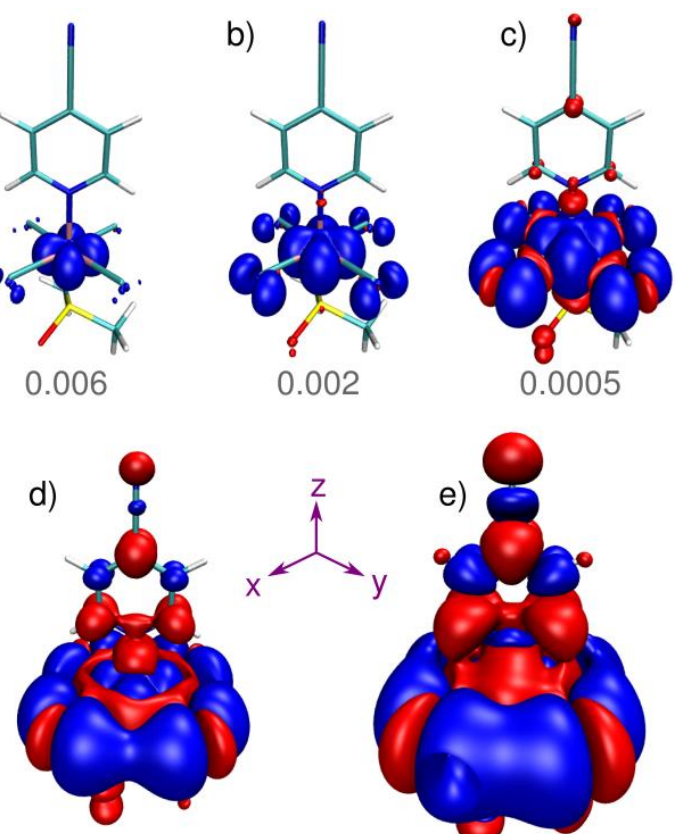

0.0001

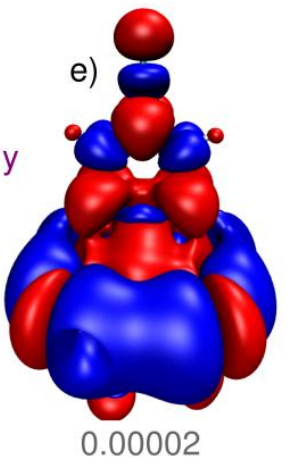

a)

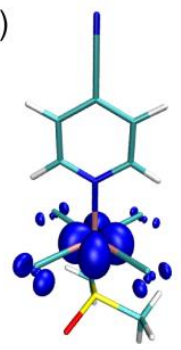

0.006

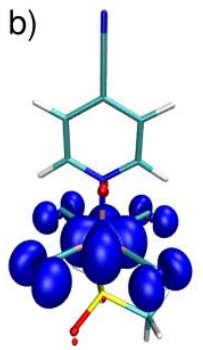

0.002
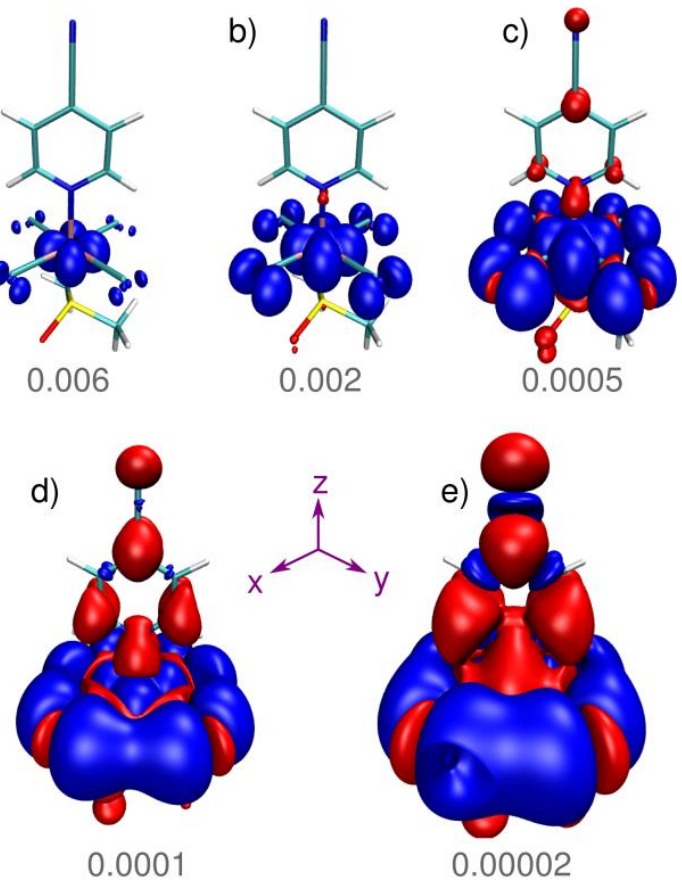

Figure S6. Visualization of the $z$-component of the spin density calculated at the $4 \mathrm{c}$ relativistic level using the PBE0 (top) or PBE (bottom) functional for compound 3. 


\section{DISCUSSION}

Table S1. Experimental (X-ray) and calculated (DFT) $\mathrm{Ru}-\mathrm{N}$ and $\mathrm{Ru}-\mathrm{S}$ interatomic distances for compounds $\mathbf{2}$ and $\mathbf{3}$. The calculated geometries were optimized at the PBE0/COSMO level using various basis sets (def2-TZVPP, def2-QZVPP, def2-QZVPP-D3).

\begin{tabular}{ccccccccc}
\hline & $\mathbf{2}$ & \multicolumn{7}{c}{$\mathbf{3}$} \\
& $\mathbf{e x p}$ & TZVPP & QZVPP & QZVPP -D3 & exp & TZVPP & QZVPP & QZVPP -D3 \\
\hline Ru-N & $\mathbf{2 . 1 1 3}$ & 2.114 & 2.107 & 2.099 & $\mathbf{2 . 1 2 8}$ & 2.110 & 2.104 & 2.096 \\
Ru-S & $\mathbf{2 . 2 9 1}$ & 2.292 & 2.278 & 2.269 & $\mathbf{2 . 2 7 8}$ & 2.292 & 2.279 & 2.271 \\
\hline
\end{tabular}

Table S2. The orbital contributions ( $\delta^{\text {orb }}$ ) to the ${ }^{1} \mathrm{H}$ and ${ }^{13} \mathrm{C}$ NMR chemical shifts for compounds $\mathbf{2}$ and $\mathbf{3}$ calculated at the SO-ZORA/PBE0/COSMO level by using basis set TZ2P or QZ4P. Two different geometries (def2-TZVPP and def2-QZVPP) from Table S1 were used.

\begin{tabular}{lcccccccccc}
\hline & $\mathbf{2}$ & & \multicolumn{7}{c}{$\mathbf{3}$} \\
Geom. & exp & TZVPP & QZVPP & TZVPP & QZVPP & exp & TZVPP & QZVPP & TZVPP & QZVPP \\
NMR & & TZ2P & TZ2P & QZ4P & QZ4P & & TZ2P & TZ2P & QZ4P & QZ4P \\
\hline H2 & $\mathbf{6 . 6}$ & 8.96 & 8.92 & 8.82 & 8.81 & $\mathbf{7 . 1}$ & 9.27 & 9.23 & 9.20 & 9.17 \\
H3 & $\mathbf{7 . 4}$ & 7.02 & 7.00 & 6.96 & 6.94 & $\mathbf{7 . 6}$ & 7.28 & 7.25 & 7.27 & 7.25 \\
C2 & $\mathbf{1 6 2}$ & 155.3 & 154.9 & 155.1 & 154.7 & $\mathbf{1 6 5}$ & 156.6 & 156.2 & 157.0 & 156.6 \\
C3 & $\mathbf{1 2 3}$ & 122.6 & 122.4 & 122.2 & 122.0 & $\mathbf{1 2 3}$ & 123.7 & 123.4 & 123.8 & 123.6 \\
C4 & $\mathbf{1 5 2}$ & 148.8 & 148.6 & 148.7 & 148.5 & $\mathbf{1 2 3}$ & 114.9 & 114.5 & 114.3 & 114.0 \\
\hline
\end{tabular}


Table S3. Experimental ${ }^{1} \mathrm{H}$ and ${ }^{13} \mathrm{C}$ NMR chemical shifts for rhodium complexes $\mathbf{1 b}-\mathbf{4 b}$, and $\mathbf{6 b}$.

\begin{tabular}{|c|c|c|c|c|c|c|c|c|c|c|}
\hline & & b - H & & - Me & & - $\mathbf{C N}$ & & $-\mathrm{CF}_{3}$ & & $-\mathbf{P h}$ \\
\hline & $\mathrm{A}^{-}$ & $\mathrm{BH}^{+}$ & $A^{-}$ & $\mathrm{BH}^{+}$ & $A^{-}$ & $\mathrm{BH}^{+}$ & $\mathrm{A}^{-}$ & $\mathrm{BH}^{+}$ & $\mathrm{A}^{-}$ & $\mathrm{BH}^{+}$ \\
\hline $\mathrm{Me}_{\mathrm{DMSO}}$ & 3.6 & - & 3.6 & - & 3.6 & - & 3.6 & - & 3.6 & - \\
\hline $\mathrm{H} 2$ & 9.6 & 9.3 & 9.2 & 9.0 & 9.7 & 8.9 & 9.9 & 9.2 & 9.5 & 9.2 \\
\hline $\mathrm{H} 3$ & 7.7 & 8.4 & 7.3 & 8.1 & 8.1 & 8.0 & 8.2 & 8.1 & 7.9 & 8.6 \\
\hline H6 & - & - & - & - & - & - & - & - & 8.0 & 8.1 \\
\hline $\mathrm{H} 7$ & - & - & - & - & - & - & - & - & 7.6 & 7.7 \\
\hline $\mathrm{H}_{\text {subst. }}$ & 8.2 & 8.9 & 2.5 & 2.7 & - & - & - & - & 7.6 & 7.7 \\
\hline $\mathrm{Me}_{\mathrm{DMSO}}$ & 45 & - & 45 & - & 45 & - & 45 & - & 45 & - \\
\hline $\mathrm{C} 2$ & 156 & 143 & 156 & 143 & 157 & 152 & 157 & 151 & 157 & 144 \\
\hline C3 & 123 & 128 & 125 & 129 & 126 & 127 & 119 & 120 & 122 & 125 \\
\hline $\mathrm{C} 4$ & 138 & 146 & 151 & 161 & 122 & 121 & 139 & 138 & 150 & 157 \\
\hline C5 & - & - & - & - & - & - & - & - & 138 & 136 \\
\hline C6 & - & - & - & - & - & - & - & - & 128 & 129 \\
\hline C7 & - & - & - & - & - & - & - & - & 131 & 133 \\
\hline C8 & - & - & - & - & - & - & - & - & 130 & 131 \\
\hline $\mathrm{C}_{\text {subst. }}$ & - & - & 21 & 23 & 117 & 118 & 123 & 123 & - & - \\
\hline N1 & 238 & * & 229 & 206 & 254 & 258 & $-^{\mathrm{a}}$ & $-^{a}$ & 230 & 209 \\
\hline
\end{tabular}




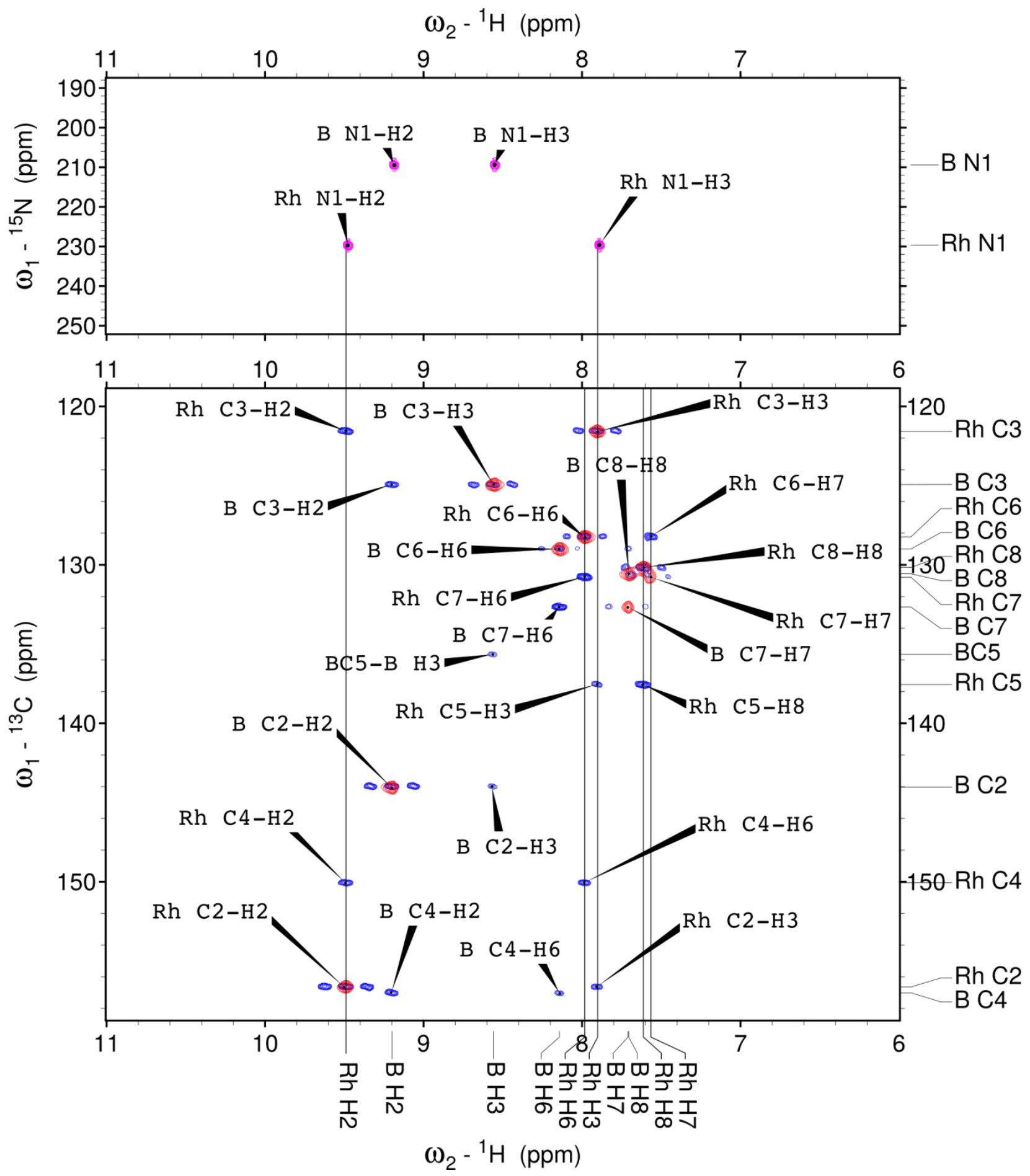

Figure S7. ${ }^{1} \mathrm{H}^{-15} \mathrm{~N}$ HMBC (top, magenta, ${ }^{n} J_{\mathrm{H}-\mathrm{N}}=5 \mathrm{~Hz}$ ) spectrum and overlaid ${ }^{1} \mathrm{H}_{-}-{ }^{13} \mathrm{C}$ HSQC (red, $\left.{ }^{1} J_{\mathrm{H}-\mathrm{C}}=145 \mathrm{~Hz}\right)$ and ${ }^{1} \mathrm{H}_{-}{ }^{13} \mathrm{C}$ HMBC $\left(\right.$ blue,$\left.{ }^{\mathrm{n}} \mathrm{J}_{\mathrm{H}-\mathrm{C}}=10 \mathrm{~Hz}\right)$ spectra of compound $6 \mathbf{b}$ recorded in DMF- $d_{7}$ at $293 \mathrm{~K}$. Rh and $\mathbf{B}$ denote the rhodium diamagnetic complex and the counter-cation (corresponding protonated base), respectively. 


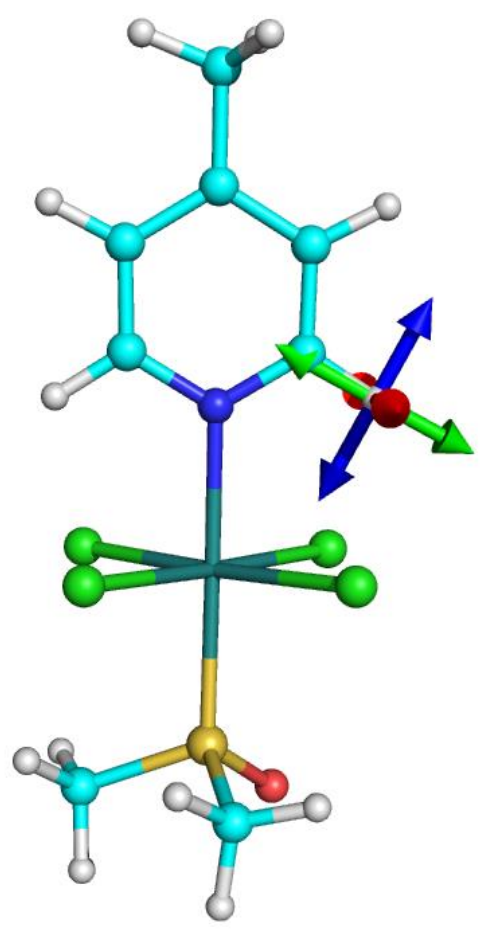

Figure S8. The orientations of the principal axes of the $A$-tensor ( $A_{\mathrm{xx}}$ in red, $A_{\mathrm{yy}}$ in green, $A_{\mathrm{zz}}$ in blue) for the $\mathrm{H} 2$ atom in compound 2, calculated at the PBE0/TZ2P/SO-ZORA level in the COSMO implicit model of solvent (DMF). 
Table S4. The calculated ${ }^{1} \mathrm{H}$ NMR chemical shifts (PBE0/TZ2P/SO-ZORA) decomposed into orbital contribution, contact and pseudocontact terms in vacuum and using the COSMO implicit model of the solvent (DMF).

\begin{tabular}{|c|c|c|c|c|c|c|c|c|c|c|c|c|c|c|c|c|c|}
\hline & & \multicolumn{2}{|c|}{1} & \multicolumn{2}{|c|}{2} & \multicolumn{2}{|c|}{3} & \multicolumn{2}{|c|}{4} & \multicolumn{2}{|l|}{5} & \multicolumn{2}{|c|}{6} & \multicolumn{2}{|c|}{7} & \multicolumn{2}{|c|}{8} \\
\hline & & vac & sol & vac & sol & vac & sol & vac & sol & vac & sol & vac & sol & vac & sol & vac & sol \\
\hline \multirow[t]{3}{*}{$\mathrm{H} 2$} & $\delta^{\text {orb }}$ & +9.5 & +9.0 & +9.6 & +9.0 & +9.6 & $\begin{array}{l}+9.3 \\
\end{array}$ & 10.0 & +9.3 & +9.6 & +9.2 & +9.5 & +9.1 & +9.5 & +9.3 & 10.7 & +9.1 \\
\hline & $\delta^{\mathrm{con}}$ & -0.1 & -6.4 & -0.6 & -6.9 & +4.6 & -3.6 & +2.4 & -4.3 & +4.0 & -3.9 & +0.9 & -6.0 & +0.7 & -6.1 & +2.7 & -5.6 \\
\hline & $\delta^{\mathrm{pc}}$ & -5.4 & -7.7 & -5.5 & -8.0 & -5.1 & -7.2 & -5.2 & -7.0 & -4.9 & -7.0 & -5.1 & -7.5 & -5.2 & -7.5 & -5.0 & -7.3 \\
\hline \multirow{3}{*}{$\mathrm{H} 3$} & $\delta^{\text {orb }}$ & +6.0 & +7.2 & +5.8 & +7.0 & +5.6 & +7.3 & +6.4 & +7.4 & +5.8 & +7.5 & +5.5 & +7.5 & +5.5 & +7.7 & +7.7 & +7.5 \\
\hline & $\delta^{\mathrm{con}}$ & -9.3 & -10.1 & -9.2 & -10.0 & -9.7 & -9.9 & -9.3 & -9.6 & -9.4 & -9.7 & -9.3 & -9.9 & -9.3 & -9.9 & -9.5 & -9.9 \\
\hline & $\delta^{\mathrm{pc}}$ & +0.1 & +0.1 & +0.3 & +0.3 & -0.4 & 0.0 & 0.0 & +0.4 & -0.2 & +0.3 & 0.0 & +0.3 & 0.0 & +0.3 & 0.0 & +0.3 \\
\hline \multirow{3}{*}{$\mathrm{H} 4 / \mathrm{Me}$} & $\delta^{\text {orb }}$ & +6.3 & +7.5 & +1.2 & +1.9 & - & - & - & - & - & - & - & - & - & - & - & - \\
\hline & $\delta^{\mathrm{con}}$ & +6.8 & +1.5 & -8.5 & -5.1 & - & - & - & - & - & - & - & - & - & - & - & - \\
\hline & $\delta^{\mathrm{pc}}$ & -1.0 & -1.4 & -0.7 & -1.0 & - & - & - & - & - & - & - & - & - & - & - & - \\
\hline \multirow{3}{*}{ H6 } & $\delta^{\text {orb }}$ & - & - & - & - & - & - & - & - & - & - & +6.8 & +7.8 & +6.8 & +7.9 & +8.1 & +7.8 \\
\hline & $\delta^{\mathrm{con}}$ & - & - & - & - & - & - & - & - & - & - & +1.4 & -0.1 & +1.4 & 0.0 & +2.0 & 0.0 \\
\hline & $\delta^{\mathrm{pc}}$ & - & - & - & - & - & - & - & - & - & - & -0.7 & -1.0 & -0.7 & -1.0 & -1.0 & -1.0 \\
\hline \multirow[t]{3}{*}{ H7 } & $\delta^{\text {orb }}$ & - & - & - & - & - & - & - & - & - & - & +6.3 & +7.4 & +6.3 & +7.6 & +7.4 & +7.7 \\
\hline & $\delta^{\mathrm{con}}$ & - & - & - & - & - & - & - & - & - & - & -1.5 & -0.8 & -1.5 & -0.8 & -1.6 & -0.9 \\
\hline & $\delta^{\mathrm{pc}}$ & - & - & - & - & - & - & - & - & - & - & 0.0 & -0.4 & 0.0 & -0.4 & -0.2 & -0.4 \\
\hline \multirow[t]{3}{*}{$\mathrm{H} 8 / \mathrm{Me}$} & $\delta^{\text {orb }}$ & - & - & - & - & - & - & - & - & - & - & +7.6 & +7.3 & +1.6 & +2.6 & - & - \\
\hline & $\delta^{\mathrm{con}}$ & - & - & - & - & - & - & - & - & - & - & +2.0 & +0.4 & -2.3 & -1.1 & - & - \\
\hline & $\delta^{\mathrm{pc}}$ & - & - & - & - & - & - & - & - & - & - & -0.2 & -0.3 & -0.2 & -0.2 & - & - \\
\hline \multirow[t]{3}{*}{$\mathrm{Me}_{\mathrm{DMSO}}$} & $\delta^{\text {orb }}$ & +3.1 & +3.4 & +3.1 & +3.4 & +2.9 & +3.4 & +3.4 & +3.7 & +3.0 & +3.4 & +2.9 & +3.4 & +2.9 & +3.7 & +3.7 & +3.7 \\
\hline & $\delta^{\mathrm{con}}$ & -10.7 & -12.8 & -8.5 & -12.8 & -10.9 & -12.9 & -10.8 & -12.9 & -10.8 & -12.8 & -10.7 & -12.8 & -10.7 & -12.7 & -10.7 & -12.8 \\
\hline & $\delta^{\mathrm{pc}}$ & -2.9 & -4.2 & -0.7 & -4.3 & -2.8 & -4.0 & -2.8 & -3.9 & -2.7 & -3.9 & -2.8 & -4.1 & -2.8 & -4.1 & -2.8 & -4.0 \\
\hline
\end{tabular}


Table S5. Calculated ${ }^{13} \mathrm{C}$ NMR chemical shifts (PBE0/TZ2P/SO-ZORA) decomposed into orbital contribution, contact and pseudocontact terms in vacuum and using the COSMO implicit model of the solvent (DMF).

\begin{tabular}{|c|c|c|c|c|c|c|c|c|c|c|c|c|c|c|c|c|c|}
\hline & & \multicolumn{2}{|l|}{1} & \multicolumn{2}{|c|}{2} & \multicolumn{2}{|c|}{3} & \multicolumn{2}{|c|}{4} & \multicolumn{2}{|c|}{5} & \multicolumn{2}{|c|}{6} & \multicolumn{2}{|c|}{7} & \multicolumn{2}{|l|}{8} \\
\hline & & vac & sol & vac & sol & vac & sol & vac & sol & vac & sol & vac & sol & vac & sol & vac & sol \\
\hline \multirow[t]{3}{*}{$\mathrm{C} 2$} & $\delta^{\text {orb }}$ & +160 & +156 & +161 & +155 & +163 & +157 & +164 & +157 & +165 & +156 & +167 & +156 & +167 & +158 & +159 & +156 \\
\hline & $\delta^{\text {con }}$ & -84 & -75 & -83 & -74 & -97 & -84 & -92 & -80 & -96 & -82 & -87 & -76 & -87 & -76 & -92 & -77 \\
\hline & $\delta^{\mathrm{pc}}$ & -1 & -1 & -1 & -1 & -2 & -2 & -1 & -1 & -2 & -2 & -1 & -1 & -1 & -1 & -1 & -1 \\
\hline \multirow[t]{3}{*}{ C3 } & $\delta^{\text {orb }}$ & +113 & +122 & +113 & +123 & +117 & +124 & +110 & +117 & +115 & +119 & +115 & +119 & +115 & +121 & +98 & +119 \\
\hline & $\delta^{\mathrm{con}}$ & -6 & -23 & -7 & -26 & +19 & -14 & +2 & -18 & +11 & -16 & 0 & -23 & 0 & -23 & +4 & -21 \\
\hline & $\delta^{\mathrm{pc}}$ & 0 & -2 & 0 & -2 & -1 & -1 & +1 & -1 & 0 & -1 & +1 & -2 & +1 & -2 & +2 & -2 \\
\hline \multirow[t]{3}{*}{$\mathrm{C} 4$} & $\delta^{\text {orb }}$ & +122 & +136 & +130 & +149 & +117 & +115 & +131 & +137 & +145 & +134 & +163 & +147 & +162 & +149 & +111 & +145 \\
\hline & $\delta^{\mathrm{con}}$ & -47 & -25 & -48 & -24 & -81 & -38 & -64 & -33 & -69 & -34 & -58 & -27 & -57 & -27 & -66 & -29 \\
\hline & $\delta^{\mathrm{pc}}$ & -4 & -4 & -4 & -4 & -6 & -4 & -5 & -4 & -5 & -4 & -4 & -4 & -4 & -4 & -5 & -4 \\
\hline C5/ & $\delta^{\text {orb }}$ & - & - & +13 & +13 & +130 & +124 & +127 & +122 & +175 & +167 & +152 & +139 & +149 & +137 & +132 & +145 \\
\hline \multirow{2}{*}{$\mathrm{C}_{\text {subst. }}{ }^{\mathrm{a}}$} & $\delta^{\mathrm{con}}$ & - & - & +13 & +3 & +59 & +22 & +26 & +10 & +31 & +11 & +26 & +9 & +26 & +9 & +32 & +10 \\
\hline & $\delta^{\mathrm{pc}}$ & - & - & -1 & -1 & 0 & -1 & -1 & -1 & -1 & -1 & -1 & -1 & -1 & -1 & -1 & -1 \\
\hline \multirow[t]{3}{*}{ C6 } & $\delta^{\text {orb }}$ & - & - & - & - & - & - & - & - & - & - & +125 & +127 & +125 & +129 & +125 & +127 \\
\hline & $\delta^{\mathrm{con}}$ & - & - & - & - & - & - & - & - & - & - & -19 & -8 & -19 & -8 & -23 & -10 \\
\hline & $\delta^{\mathrm{pc}}$ & - & - & - & - & - & - & - & - & - & - & -1 & -1 & -1 & -1 & -1 & -1 \\
\hline \multirow[t]{3}{*}{ C7 } & $\delta^{\text {orb }}$ & - & - & - & - & - & - & - & - & - & - & +126 & +129 & +126 & +132 & +127 & +134 \\
\hline & $\delta^{\mathrm{con}}$ & - & - & - & - & - & - & - & - & - & - & +9 & +3 & +9 & +3 & +13 & +4 \\
\hline & $\delta^{\mathrm{pc}}$ & - & - & - & - & - & - & - & - & - & - & 0 & 0 & 0 & 0 & 0 & 0 \\
\hline \multirow[t]{3}{*}{$\mathrm{C} 8$} & $\delta^{\text {orb }}$ & - & - & - & - & - & - & - & - & - & - & +128 & +129 & +137 & +144 & +74 & +108 \\
\hline & $\delta^{\mathrm{con}}$ & - & - & - & - & - & - & - & - & - & - & -12 & -5 & -13 & -5 & -18 & -6 \\
\hline & $\delta^{\mathrm{pc}}$ & - & - & - & - & - & - & - & - & - & - & -1 & -1 & -1 & -1 & -1 & -1 \\
\hline \multirow[t]{3}{*}{$\mathrm{C}_{\text {subst. }}{ }^{\mathrm{a}}$} & $\delta^{\text {orb }}$ & - & - & - & - & - & - & - & - & - & - & - & - & +25 & +20 & +128 & +124 \\
\hline & $\delta^{\mathrm{con}}$ & - & - & - & - & - & - & - & - & - & - & - & - & +3 & +2 & +14 & +3 \\
\hline & $\delta^{\mathrm{pc}}$ & - & - & - & - & - & - & - & - & - & - & - & - & 0 & 0 & 0 & 0 \\
\hline \multirow[t]{3}{*}{$\mathrm{Me}_{\text {DMSO }}$} & $\delta^{\text {orb }}$ & +40 & +43 & +40 & +42 & +36 & +43 & +38 & +40 & +41 & +43 & +40 & +43 & +40 & +40 & +43 & +40 \\
\hline & $\delta^{\mathrm{con}}$ & -186 & -157 & -187 & -158 & -182 & -155 & -181 & -156 & -182 & -156 & -185 & -157 & -186 & -158 & -184 & -157 \\
\hline & $\delta^{\mathrm{pc}}$ & -4 & -5 & -4 & -5 & -4 & -5 & -4 & -4 & -4 & -4 & -4 & -5 & -4 & -5 & -4 & -5 \\
\hline
\end{tabular}

${ }^{a} \mathrm{C}_{\text {subst. }}$ stands for the carbon atom of the $\mathrm{Me}, \mathrm{CN}, \mathrm{CF}_{3}$, or $\mathrm{COOH}$ group. 
Table S6. Calculated ${ }^{1} \mathrm{H}$ and ${ }^{13} \mathrm{C}$ NMR chemical shifts for rhodium complexes $\mathbf{1 b}-\mathbf{4 b}$, and $\mathbf{6 b}$.

\begin{tabular}{cccccc}
\hline & $\mathbf{1 b}-\mathbf{H}$ & $\mathbf{2 b}-\mathbf{M e}$ & $\mathbf{3 b}-\mathbf{C N}$ & $\mathbf{4 b}-\mathbf{C F}_{\mathbf{3}}$ & $\mathbf{6 b}-\mathbf{P h}$ \\
\hline $\mathrm{Me}_{\text {DMSO }}$ & 3.7 & 3.5 & 3.9 & 3.8 & 3.8 \\
$\mathrm{H} 2$ & 8.9 & 8.7 & 9.2 & 9.0 & 8.8 \\
$\mathrm{H} 3$ & 7.2 & 7.1 & 7.7 & 7.6 & 7.6 \\
$\mathrm{H} 6$ & - & - & - & - & 7.8 \\
$\mathrm{H} 7$ & - & - & - & - & 7.4 \\
$\mathrm{H}_{\text {subst. }}$ & 7.7 & 2.0 & - & - & 7.3 \\
$\mathrm{Me}_{\text {DMSO }}$ & 41 & 45 & 49 & 42 & 42 \\
$\mathrm{C} 2$ & 155 & 154 & 157 & 156 & 154 \\
$\mathrm{C} 3$ & 124 & 125 & 129 & 120 & 121 \\
$\mathrm{C} 4$ & 141 & 155 & 123 & 141 & 151 \\
$\mathrm{C} 5$ & - & - & - & - & 137 \\
$\mathrm{C} 6$ & - & - & - & - & 128 \\
$\mathrm{C} 7$ & - & - & - & - & 129 \\
$\mathrm{C} 8$ & - & - & - & - & 131 \\
$\mathrm{C}_{\text {subst. }}$ & - & 13 & 122 & 121 & - \\
$\mathrm{N} 1$ & 241 & 230 & 253 & 253 & 234 \\
\hline
\end{tabular}


Table S7. The experimental and calculated ${ }^{1} \mathrm{H}$ and ${ }^{13} \mathrm{C}$ NMR chemical shifts ( $\delta$ in $\left.\mathrm{ppm}\right)$ for compounds 2 and 3 in DMF solution at $293 \mathrm{~K}$. The calculated principal values of the hyperfine coupling tensors ( $\mathbf{A}$ in $\mathbf{M H z}$ ) and the $\mathbf{g}$ tensors, ${ }^{a}$ as well as the corresponding isotropic values, are also included.

\begin{tabular}{|c|c|c|c|c|c|c|c|c|c|c|c|}
\hline & & & & C3 & & & $\mathrm{C}$ & & & $\mathrm{H}$ & \\
\hline & & & 2 & 3 & $\Delta(3-2)$ & 2 & 3 & $\Delta(3-2)$ & 2 & 3 & $\Delta(3-2)$ \\
\hline$\overline{\mathrm{EXP}}$ & $\delta^{\text {orb }}$ & & +123 & +124 & +1 & +162 & +165 & +3 & +6.6 & +7.1 & +0.5 \\
\hline & $\delta^{\text {para }}$ & & -25 & -17 & +8 & -72 & -78 & -6 & -14.0 & -11.8 & +2.2 \\
\hline & $\delta^{\text {tot }}$ & & +98 & +107 & +9 & +90 & +87 & -3 & -7.4 & -4.7 & +2.7 \\
\hline $\begin{array}{l}\text { SO-ZORA } \\
\text { (COSMO) }\end{array}$ & $\delta^{\text {orb }}$ & & +123 & +124 & +1 & +155 & +157 & +2 & +9.0 & +9.3 & +0.3 \\
\hline & $\delta^{\text {para }}$ & & -28 & -15 & +13 & -75 & -86 & -11 & -14.9 & -10.8 & +4.1 \\
\hline & & $\delta^{c o n}$ & -26 & -14 & +12 & -74 & -84 & -10 & -6.9 & -3.6 & +3.3 \\
\hline & & $\delta^{p c}$ & -2 & -1 & +1 & -1 & -2 & -1 & -8.0 & -7.2 & +0.8 \\
\hline & $\delta^{\text {tot }}$ & & +95 & +109 & +14 & +80 & +71 & -9 & -5.9 & -1.5 & +4.4 \\
\hline & $A$ & $A_{x x}$ & -0.463 & -0.409 & +0.054 & -1.247 & -1.482 & +0.235 & -3.026 & -2.933 & +0.093 \\
\hline & & $\mathrm{A}_{y y}$ & -0.256 & -0.081 & +0.175 & -1.118 & -1.143 & -0.025 & -2.636 & -2.563 & +0.073 \\
\hline & & $\mathrm{A}_{\mathrm{zz}}$ & +0.079 & +0.155 & +0.076 & +0.549 & +0.555 & +0.006 & +4.989 & +5.150 & +0.161 \\
\hline & & $\mathbf{A}_{\text {iso }}$ & -0.213 & -0.111 & +0.102 & -0.605 & -0.690 & -0.085 & -0.223 & -0.116 & +0.107 \\
\hline $\begin{array}{c}\text { SO-ZORA } \\
\text { (vacuo) }\end{array}$ & & $A_{\text {iso }}$ & -0.059 & +0.155 & +0.214 & -0.688 & -0.814 & -0.126 & -0.022 & +0.154 & +0.176 \\
\hline DKS (vacuo) ${ }^{b}$ & & $A_{\text {iso }}$ & -0.044 & +0.133 & +0.177 & -0.566 & -0.649 & -0.083 & -0.114 & +0.051 & +0.165 \\
\hline & & $A_{i s o}^{F C}$ & -0.018 & +0.167 & +0.185 & -0.550 & -0.651 & -0.101 & +0.031 & +0.190 & +0.159 \\
\hline & & $A_{i S O}^{S D}$ & +0.005 & +0.005 & 0.000 & -0.039 & -0.041 & -0.002 & -0.015 & -0.014 & +0.001 \\
\hline & & $A_{\text {iSO }}^{P S O}$ & -0.030 & -0.039 & -0.009 & +0.023 & +0.041 & +0.018 & -0.130 & -0.126 & +0.004 \\
\hline
\end{tabular}

\footnotetext{
${ }^{a}$ SO-ZORA/COSMO $g$-tensor values for 2: $g_{\mathrm{xx}}=+1.9239, g_{\mathrm{yy}}=+2.3991, g_{\mathrm{zz}}=+2.4345, g_{\text {iso }}=+2.2525 ; \mathbf{3}: g_{\mathrm{xx}}=$ $+1.9338, g_{\mathrm{yy}}=+2.3741, g_{\mathrm{zz}}=+2.4193, g_{\text {iso }}=+2.2424 ; \Delta(3-2): g_{\mathrm{xx}}=+0.0099, g_{\mathrm{yy}}=-0.0250, g_{\mathrm{zz}}=-0.0152, g_{\text {iso }}=-$ 0.0101. ${ }^{\mathrm{b}}$ Note that the DKS values were obtained by using a basis set of DZ quality in contrast to the SO-ZORA data (TZP).
} 
Table S8. The individual molecular orbitals for compound 3, calculated at the 1c level in ADF (PBE0/TZ2P/ZORA). The figures were prepared by using the adfview interface. The orbitals are plotted at the isovalue of 0.03 a.u. and indexed starting from the highest occupied energy level.

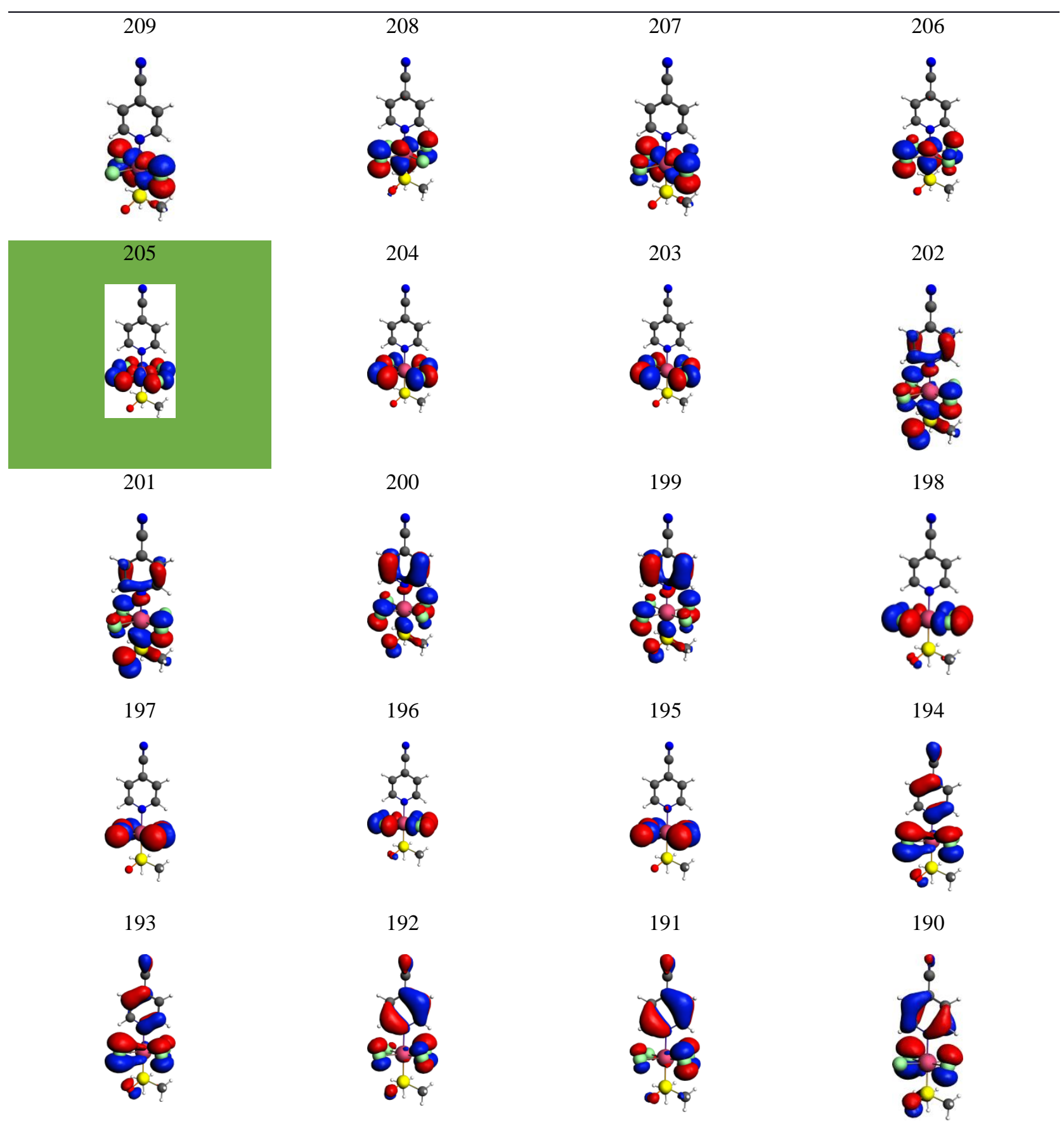




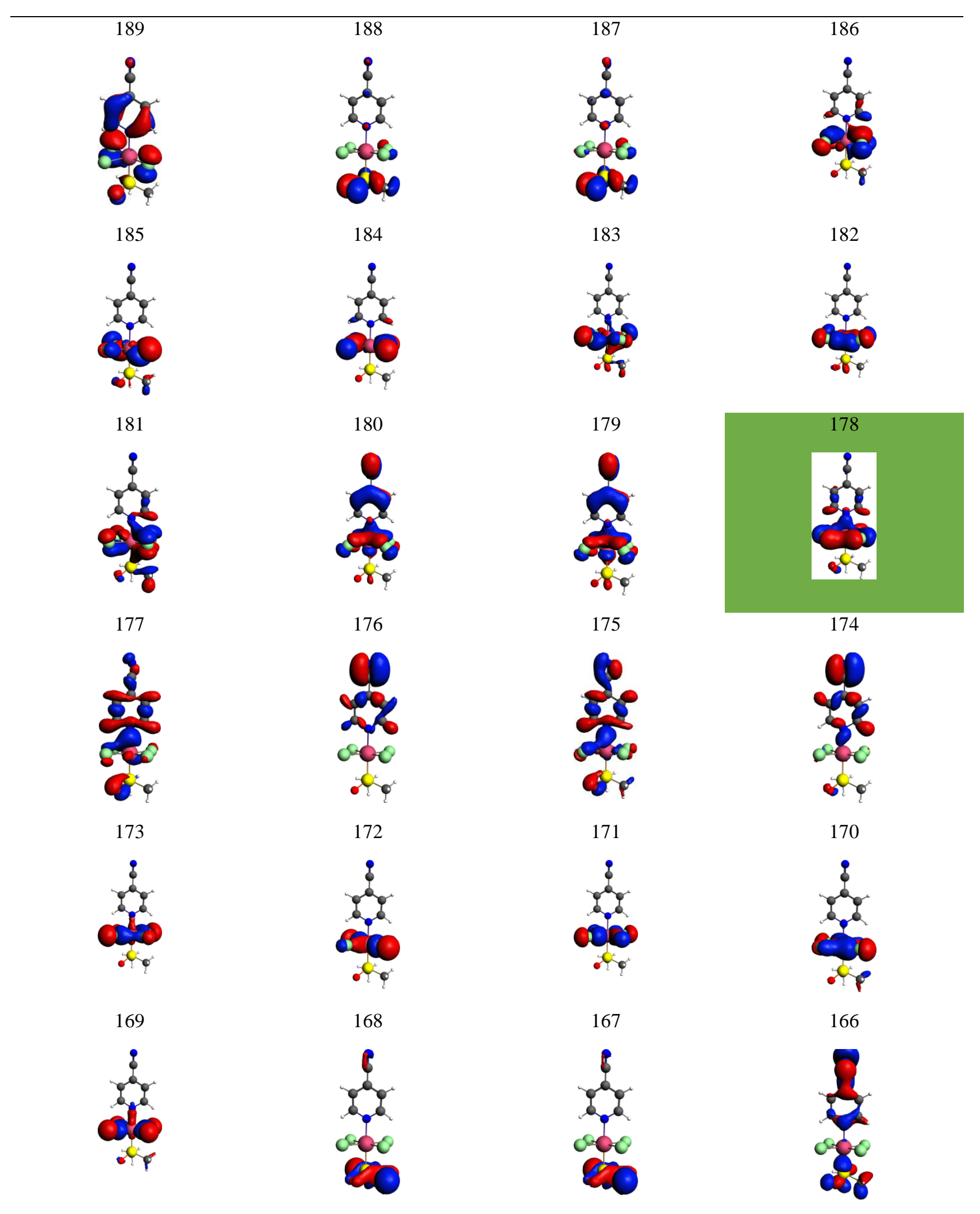




(160


a)

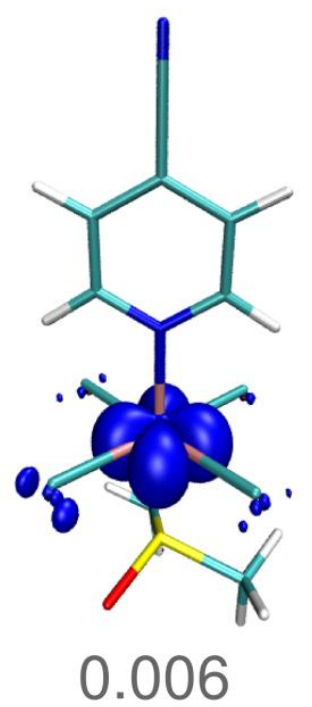

b)

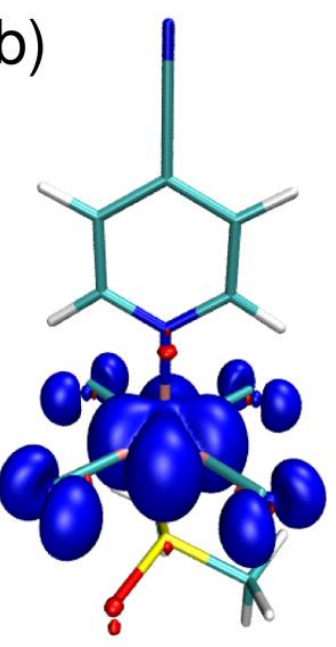

0.002

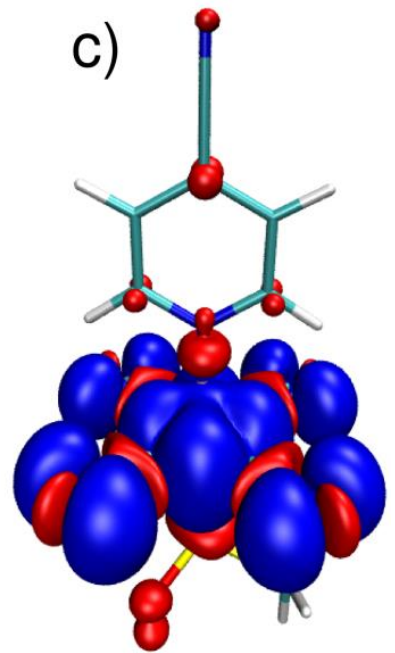

0.0005

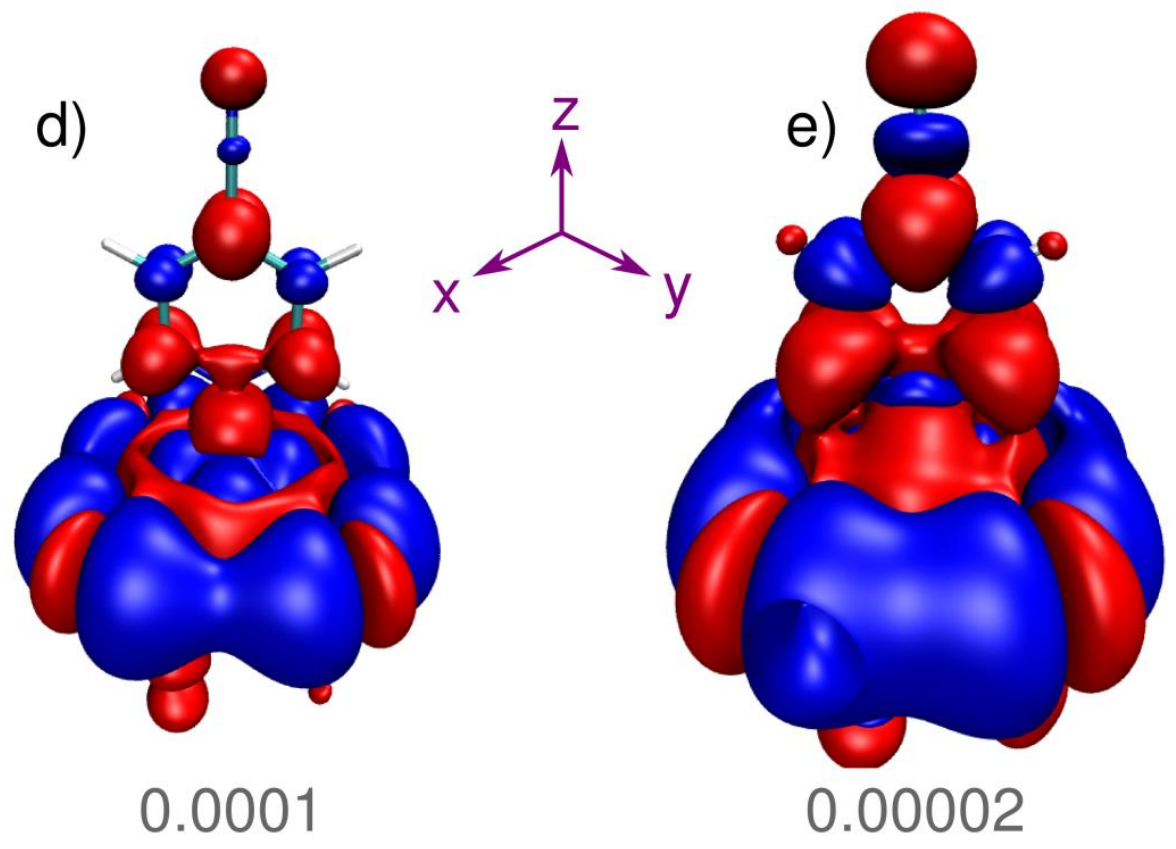

Figure S9. Visualization of the $z$-component of the spin density calculated at the $4 \mathrm{c}$ relativistic level for compound $\mathbf{3}$. 


\section{TEMPERATURE-DEPENDENT NMR SPECTRA - CURIE PLOTS}

Curie plots obtained for compounds $1,2, \mathbf{3}, \mathbf{4}, \mathbf{5}, \mathbf{6}, \mathbf{7}, \mathbf{8}$ using the program gnuplot 4.6 .4 with legends indicating the assignment and parameters of the linear fit: Coefficient of multiple determination $R^{2}=$ (SST-SSE)/SST (SST refers to the total sum of mean squared deviations, SSE to the sum of the squared deviations due to regression), $\delta_{\text {para }}$ corresponds to the paramagnetic shift at $\mathrm{T}=293 \mathrm{~K}$.
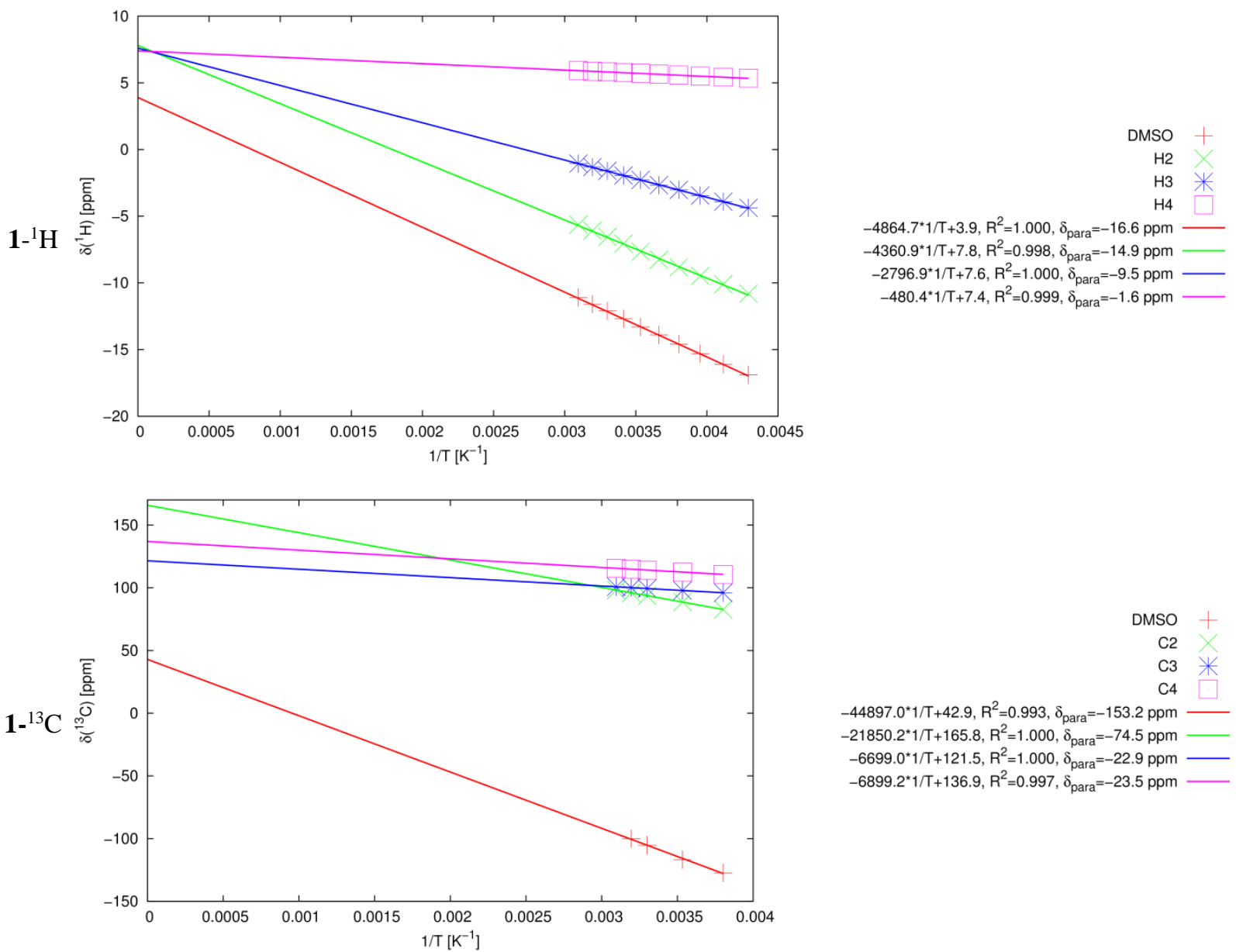


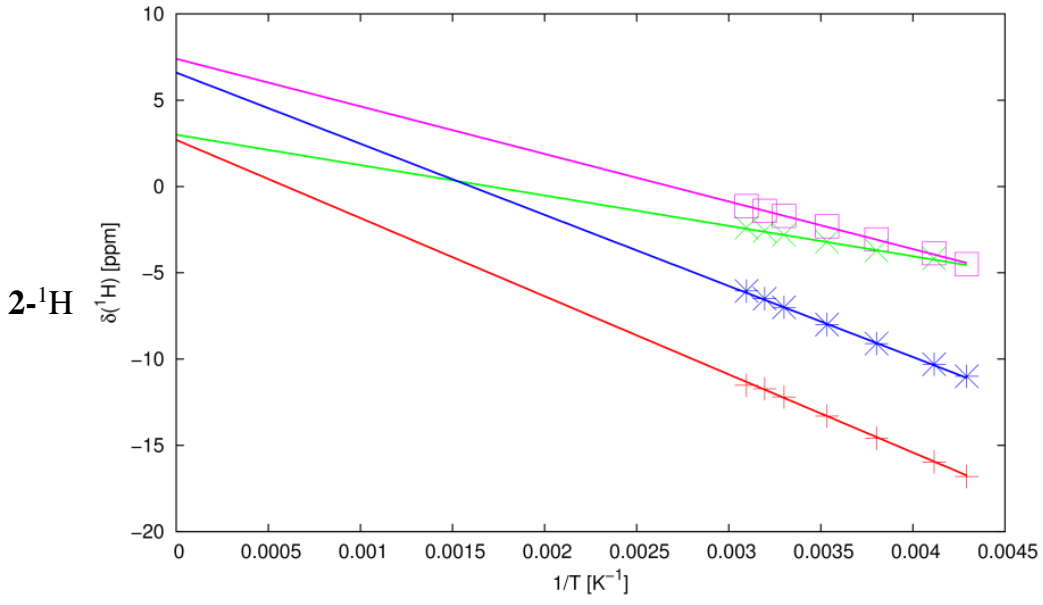

$2-{ }^{13} \mathrm{C}$
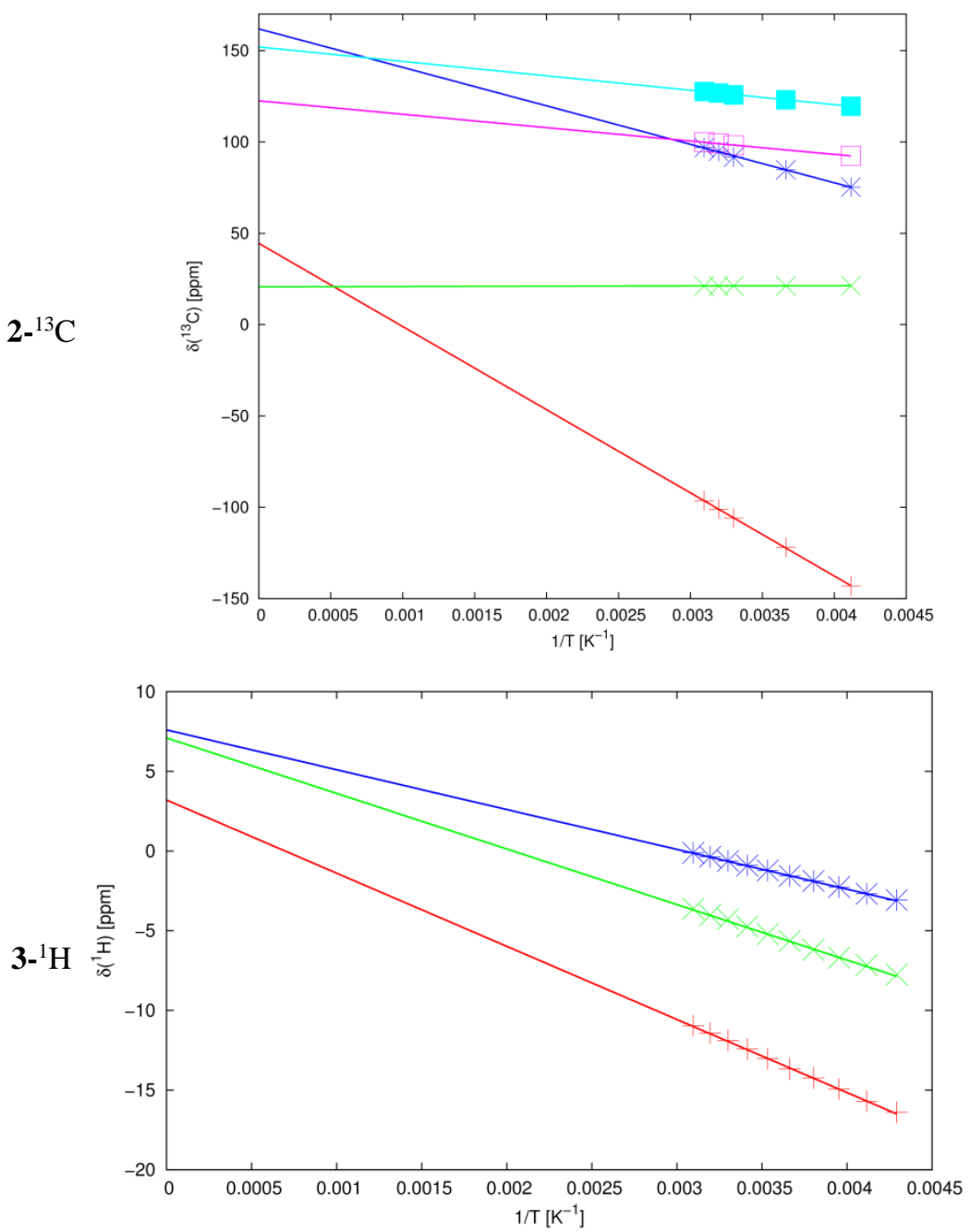

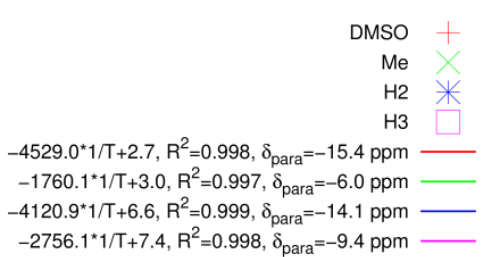

DMSO
Me
C2 $*$
C3 $\square$
C4

$+44.5, R^{2}=1.000, \delta_{\text {para }}=-155.3 \mathrm{ppm}$

$149.8^{*} 1 / T+20.7, R^{2}=0.840, \delta_{\text {para }}=0.5 \mathrm{ppm}$ $-21064.0^{*} 1 / \mathrm{T}+161.9, R^{2}=0.997, \delta_{\text {para }}=-71.9 \mathrm{ppm}$ $-7330.3^{*} 1 / T+122.5, R^{2}=0.998, \delta_{\text {para }}=-25.0 \mathrm{ppm}$ $-7896.0^{*} 1 / T+152.0, R^{2}=0.999, \delta_{\text {para }}=-26.9 \mathrm{ppm}$

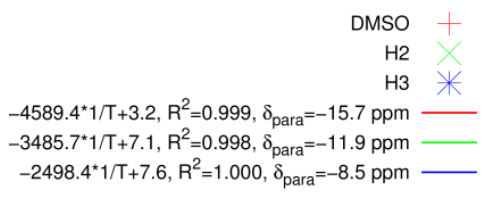



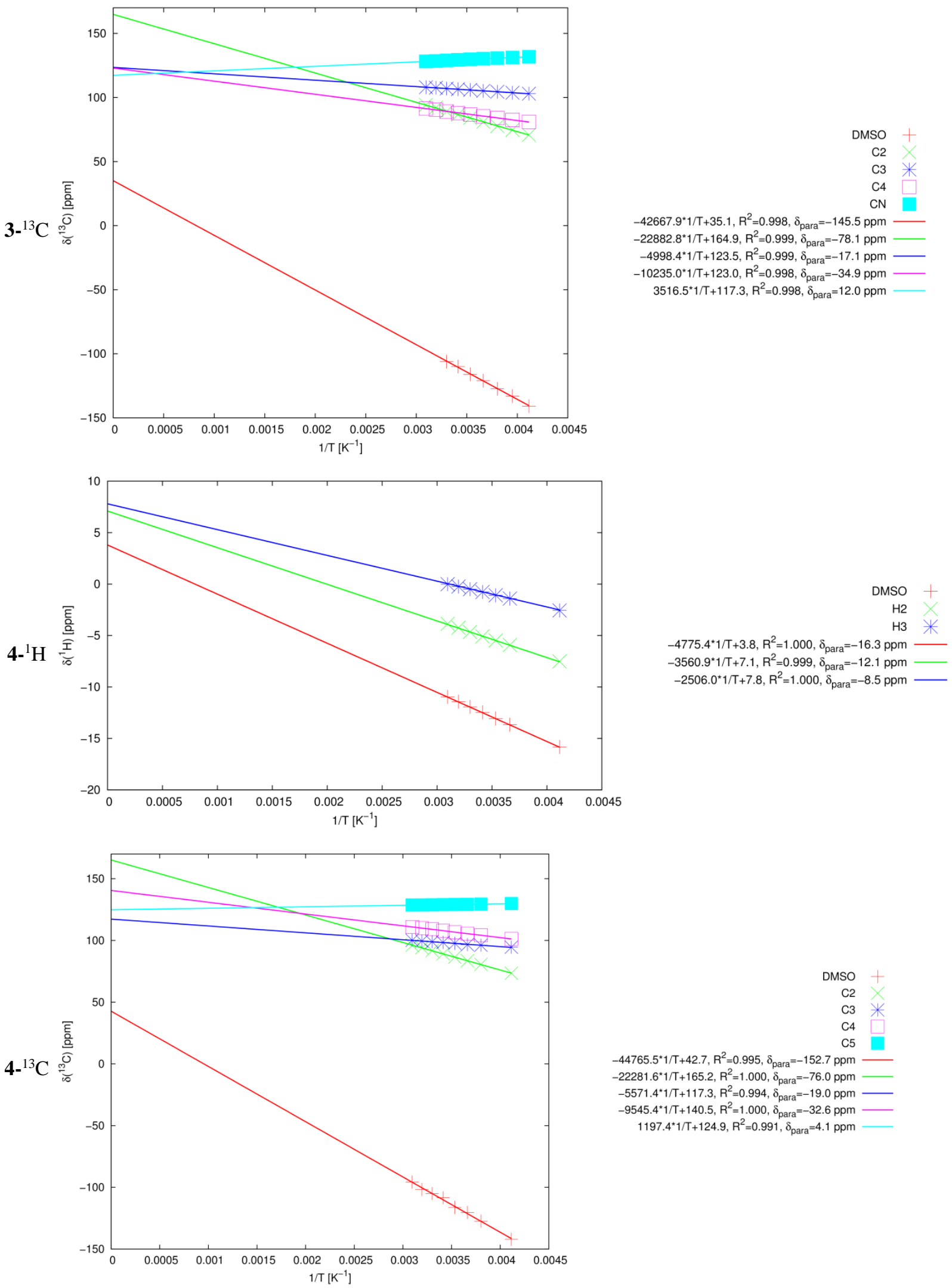

DMSO

$\mathrm{C} 2$

C3 $*$

C4

$-44765.5^{*} 1 / T+42.7, R^{2}=0.995, \delta_{\text {para }}=-152.7 \mathrm{ppm}$ $-22281.6^{*} 1 / T+165.2, R^{2}=1.000, \delta_{\text {para }}=-76.0 \mathrm{ppm}$ $-5571.4^{*} 1 / T+117.3, R^{2}=0.994, \delta_{\text {para }}=-19.0 \mathrm{ppm}$ $-9545.4^{*} 1 / T+140.5, R^{2}=1.000, \delta_{\text {para }}=-32.6 \mathrm{ppm}$ $1197.4^{*} 1 / T+124.9, R^{2}=0.991, \delta_{\text {para }}=4.1 \mathrm{ppm}$ 

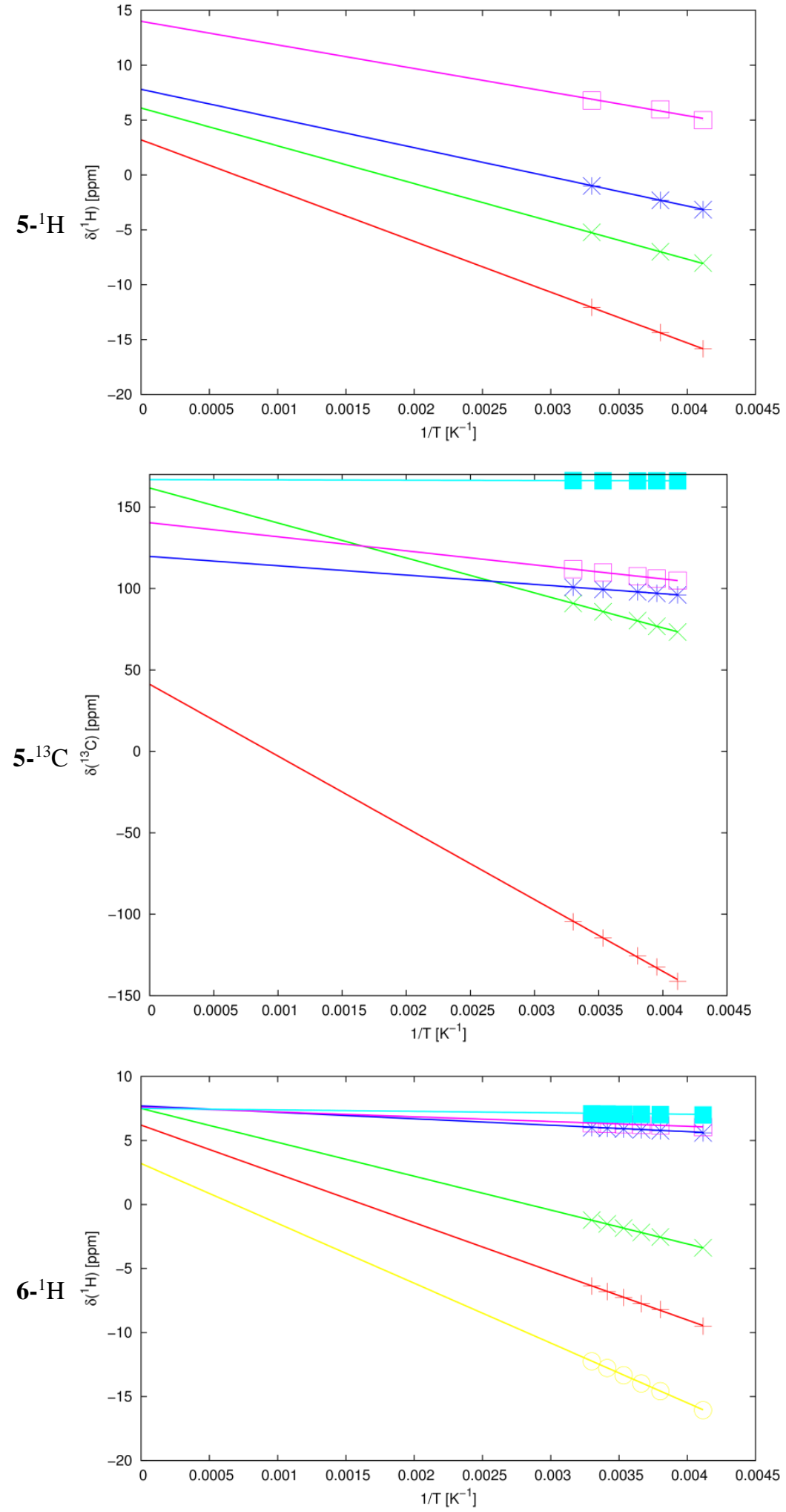
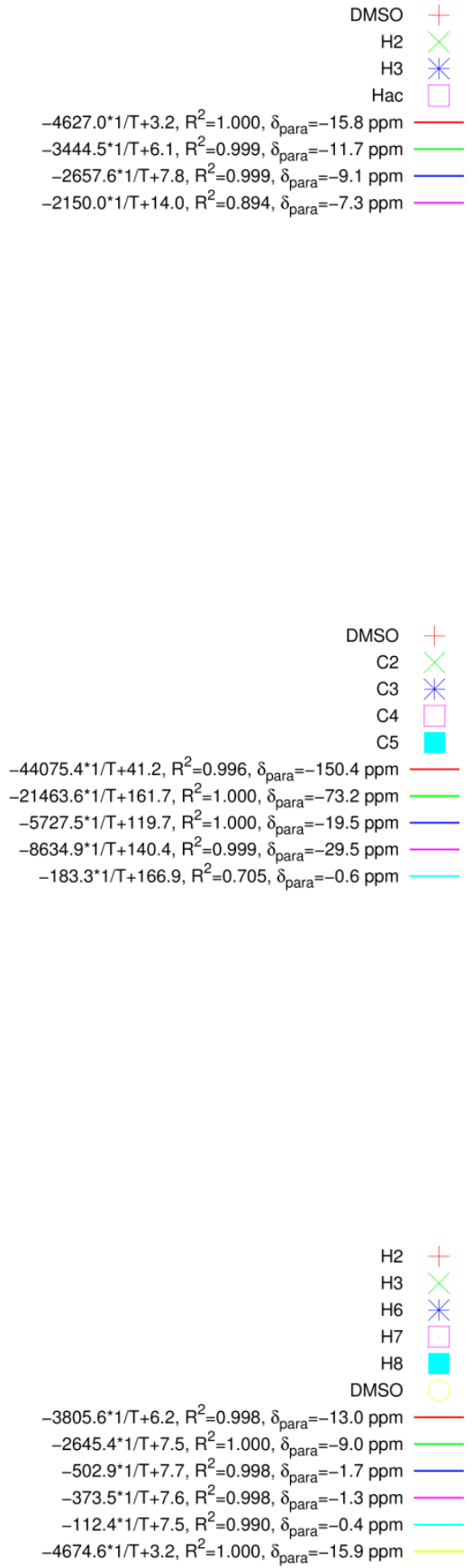

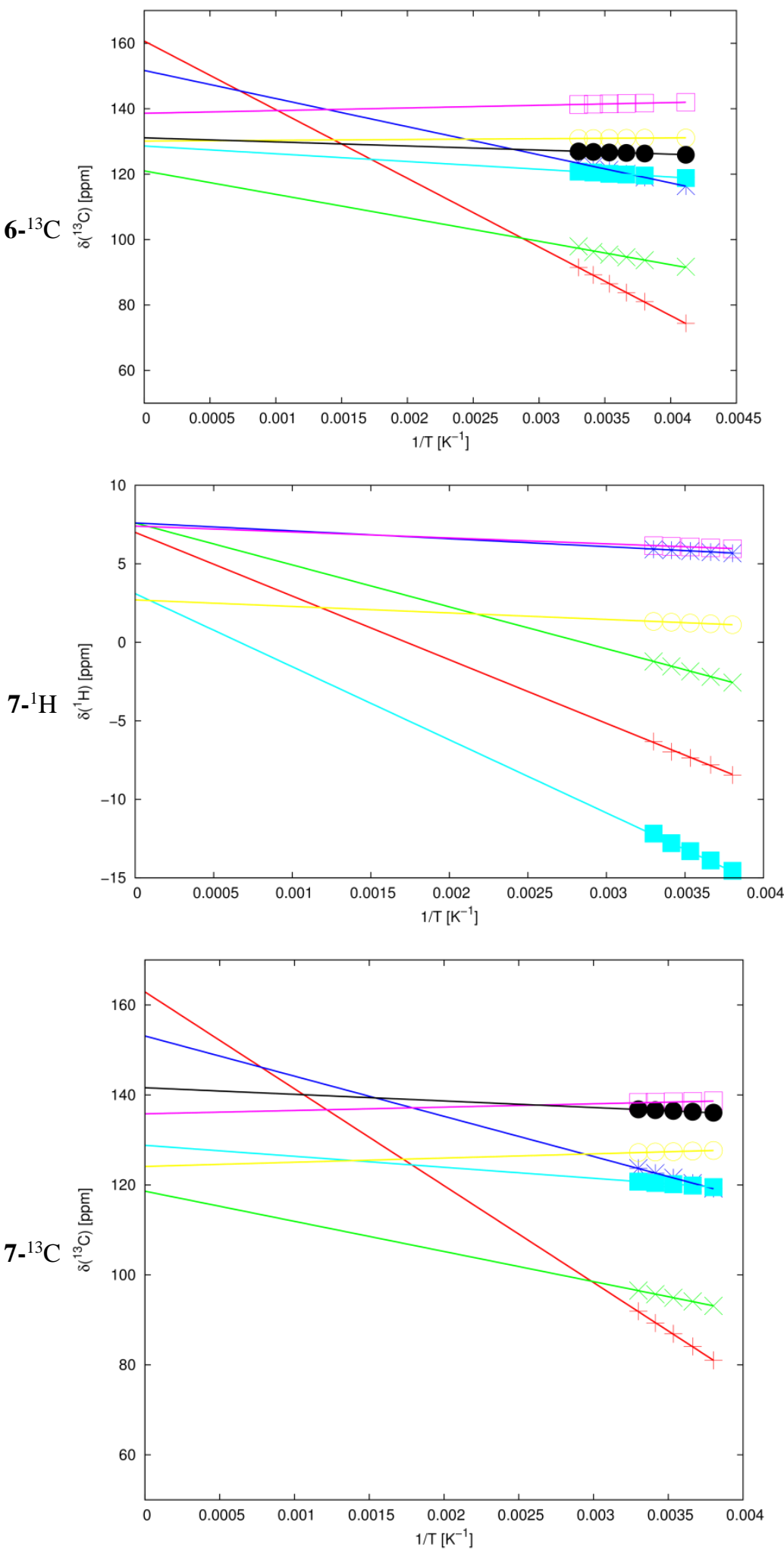
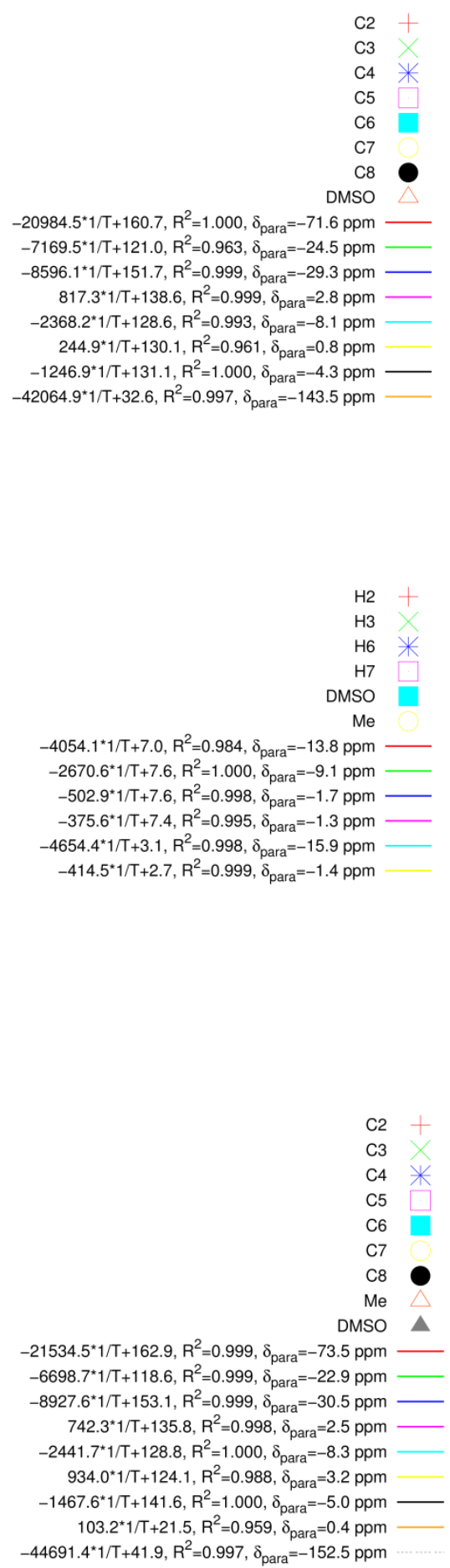

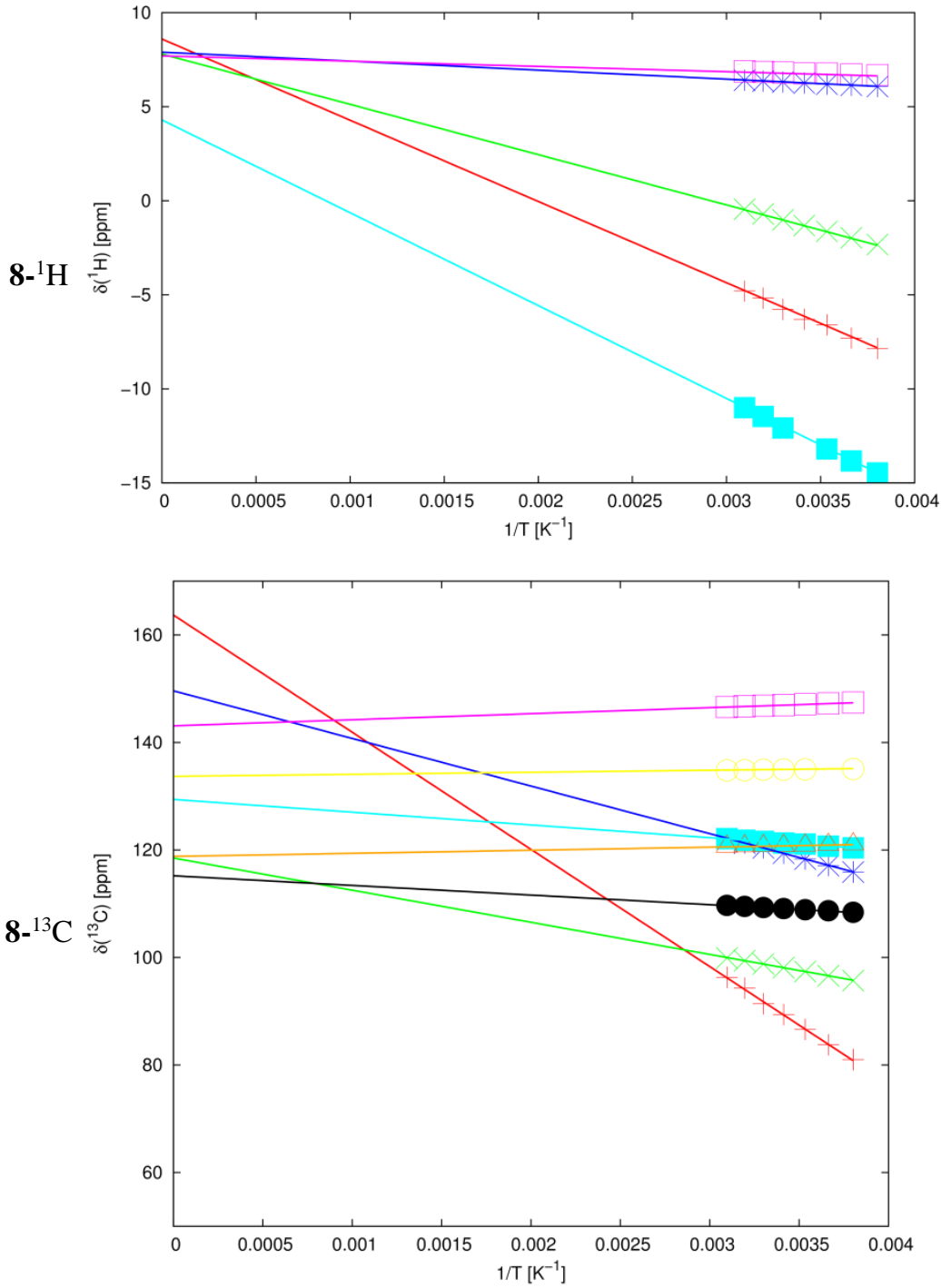
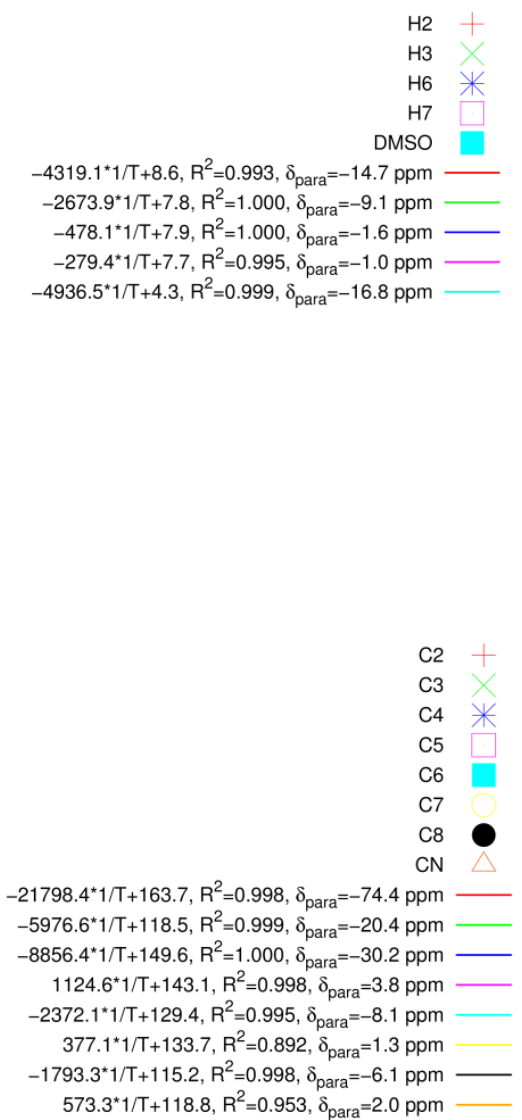


\title{
CARTESIAN COORDINATES AND ENERGIES
}

\author{
All the complexes were optimized at the PBE0/def2-TZVPP/def2-ECP/COSMO ${ }_{\text {DMF }}$ level.
}

\begin{tabular}{lrrr}
26 & \multicolumn{4}{c}{ lis } \\
COUMPOUND 1: Energy $=-2736.614903$ \\
$\mathrm{Ru}$ & 4.0892935 & 9.5160324 & 1.2362754 \\
$\mathrm{Cl}$ & 4.3221522 & 7.4934721 & 2.4228832 \\
$\mathrm{Cl}$ & 1.9716599 & 9.8023034 & 2.2032259 \\
$\mathrm{Cl}$ & 3.8603333 & 11.5892509 & 0.1419279 \\
$\mathrm{Cl}$ & 6.2515777 & 9.2573292 & 0.3256788 \\
$\mathrm{~S}$ & 3.2235089 & 8.4146764 & -0.5767935 \\
$\mathrm{O}$ & 1.7643874 & 8.2208831 & -0.6724540 \\
$\mathrm{~N}$ & 4.9255320 & 10.5344216 & 2.8910608 \\
$\mathrm{C}$ & 6.0235434 & 10.0661621 & 3.4930824 \\
$\mathrm{C}$ & 4.3785762 & 11.6655449 & 3.3483705 \\
$\mathrm{C}$ & 4.9084513 & 12.3652869 & 4.4163635 \\
$\mathrm{C}$ & 6.0469430 & 11.8819305 & 5.0400805 \\
$\mathrm{C}$ & 6.6116394 & 10.7090543 & 4.5664070 \\
$\mathrm{C}$ & 3.7498005 & 9.1676011 & -2.0997581 \\
$\mathrm{C}$ & 3.9799714 & 6.8179328 & -0.7782009 \\
$\mathrm{H}$ & 3.4933462 & 12.0053562 & 2.8321428 \\
$\mathrm{H}$ & 4.4268800 & 13.2764058 & 4.7457967 \\
$\mathrm{H}$ & 6.4855145 & 12.4088435 & 5.8783515 \\
$\mathrm{H}$ & 7.5002760 & 10.2870546 & 5.0165243 \\
$\mathrm{H}$ & 6.4296080 & 9.1499967 & 3.0909236 \\
$\mathrm{H}$ & 3.2968940 & 10.1547478 & -2.1413788 \\
$\mathrm{H}$ & 3.3994808 & 8.5337483 & -2.9137119 \\
$\mathrm{H}$ & 4.8347802 & 9.2534232 & -2.0902977 \\
$\mathrm{H}$ & 3.6732712 & 6.2083760 & 0.0678122 \\
$\mathrm{H}$ & 3.6199943 & 6.4034847 & -1.7191472 \\
$\mathrm{H}$ & 5.0613845 & 6.9420816 & -0.7770650
\end{tabular}

29

COUMPOUND 2: Energy $=-2775.900214$

$\begin{array}{lrrr}\mathrm{Ru} & 6.2606791 & 10.2484536 & 3.4036938 \\ \mathrm{Cl} & 4.1224553 & 10.7155025 & 2.5565248 \\ \mathrm{Cl} & 6.0708775 & 12.0802907 & 4.8728476 \\ \mathrm{Cl} & 8.3794243 & 9.7278757 & 4.3070929 \\ \mathrm{Cl} & 6.3749641 & 8.3687491 & 1.9873315 \\ \mathrm{~S} & 7.2820886 & 11.5627677 & 1.8282238 \\ \mathrm{O} & 6.4534962 & 12.2824002 & 0.8422670 \\ \mathrm{~N} & 5.3551191 & 9.0299834 & 4.8741761 \\ \mathrm{C} & 4.2780305 & 9.4442788 & 5.5515713 \\ \mathrm{C} & 3.6707002 & 8.6762273 & 6.5222190 \\ \mathrm{C} & 4.1746703 & 7.4157647 & 6.8330257 \\ \mathrm{C} & 5.2931033 & 6.9999407 & 6.1209422 \\ \mathrm{C} & 5.8492490 & 7.8231074 & 5.1607217 \\ \mathrm{C} & 8.3508601 & 12.7649067 & 2.5869034\end{array}$




$\begin{array}{lrrr}\mathrm{C} & 8.5010859 & 10.6424859 & 0.9174431 \\ \mathrm{H} & 3.9057181 & 10.4245300 & 5.2938910 \\ \mathrm{H} & 2.8009655 & 9.0685200 & 7.0349009 \\ \mathrm{C} & 3.5404072 & 6.5610691 & 7.8795003 \\ \mathrm{H} & 5.7411192 & 6.0318610 & 6.3076012 \\ \mathrm{H} & 6.7183272 & 7.5193903 & 4.5959451 \\ \mathrm{H} & 7.7173451 & 13.4508414 & 3.1431670 \\ \mathrm{H} & 8.8852689 & 13.2756909 & 1.7866664 \\ \mathrm{H} & 9.0294462 & 12.2499655 & 3.2643760 \\ \mathrm{H} & 7.9688693 & 9.8990617 & 0.3298628 \\ \mathrm{H} & 9.0331464 & 11.3478490 & 0.2801645 \\ \mathrm{H} & 9.1675634 & 10.1525011 & 1.6249939 \\ \mathrm{H} & 4.0642995 & 5.6127392 & 7.9914710 \\ \mathrm{H} & 2.4982460 & 6.3537065 & 7.6264785 \\ \mathrm{H} & 3.5392044 & 7.0724900 & 8.8447073\end{array}$

27

COMPound 3: Energy $=-2828.785129$

$\begin{array}{lrrr}\mathrm{Ru} & 6.2271801 & 10.1948132 & 3.4884993 \\ \mathrm{Cl} & 4.0785766 & 10.6519511 & 2.6677804 \\ \mathrm{Cl} & 6.0500767 & 12.0295640 & 4.9523699 \\ \mathrm{Cl} & 8.3538621 & 9.6801721 & 4.3706683 \\ \mathrm{Cl} & 6.3264994 & 8.3070425 & 2.0852647 \\ \mathrm{~S} & 7.2256730 & 11.5030697 & 1.8930816 \\ \mathrm{O} & 6.3780109 & 12.2120434 & 0.9175016 \\ \mathrm{~N} & 5.3406047 & 8.9844927 & 4.9722114 \\ \mathrm{C} & 4.2681872 & 9.4076081 & 5.6480624 \\ \mathrm{C} & 3.6605316 & 8.6474580 & 6.6269604 \\ \mathrm{C} & 4.1900877 & 7.3947821 & 6.9160487 \\ \mathrm{C} & 5.3068774 & 6.9526575 & 6.2157562 \\ \mathrm{C} & 5.8479995 & 7.7805677 & 5.2529494 \\ \mathrm{C} & 8.2980049 & 12.7113908 & 2.6347543 \\ \mathrm{C} & 8.4336490 & 10.5795342 & 0.9726184 \\ \mathrm{H} & 3.8954277 & 10.3861965 & 5.3866842 \\ \mathrm{H} & 2.7941291 & 9.0280644 & 7.1487275 \\ \mathrm{H} & 5.7494685 & 5.9862529 & 6.4104596 \\ \mathrm{H} & 6.7140717 & 7.4820994 & 4.6818750 \\ \mathrm{H} & 7.6682935 & 13.4002084 & 3.1917273 \\ \mathrm{H} & 8.8238017 & 13.2175887 & 1.8259559 \\ \mathrm{H} & 8.9843237 & 12.2023909 & 3.3088967 \\ \mathrm{H} & 7.8958395 & 9.8302081 & 0.3978013 \\ \mathrm{H} & 8.9534196 & 11.2825582 & 0.3227615 \\ \mathrm{H} & 9.1126626 & 10.0976380 & 1.6737607 \\ \mathrm{C} & 3.5936948 & 6.5720144 & 7.9195384 \\ \mathrm{~N} & 3.1123766 & 5.9084730 & 8.7276947\end{array}$

29

COUMPOUND 4: Energy $=-3073.490294$

$\begin{array}{lrrr}\mathrm{Ru} & -1.1159747 & -0.0458524 & -0.0518134 \\ \mathrm{Cl} & -1.0239470 & -1.6063065 & -1.8013172 \\ \mathrm{Cl} & -1.0527581 & -1.8062328 & 1.5104201 \\ \mathrm{Cl} & -1.1222768 & 1.5303804 & 1.7052118\end{array}$




$\begin{array}{lrrr}\mathrm{Cl} & -1.0722783 & 1.6912023 & -1.6416195 \\ \mathrm{~S} & -3.4067051 & -0.0480164 & -0.0332820 \\ \mathrm{O} & -4.1301351 & -0.9224281 & -0.9743494 \\ \mathrm{~N} & 0.9983810 & -0.0206783 & -0.0368415 \\ \mathrm{C} & 1.6970825 & -1.1572655 & -0.0924555 \\ \mathrm{C} & 3.0793754 & -1.1797512 & -0.0754573 \\ \mathrm{C} & 3.7597386 & 0.0226881 & 0.0007601 \\ \mathrm{C} & 3.0392428 & 1.2039659 & 0.0581616 \\ \mathrm{C} & 1.6596226 & 1.1381273 & 0.0361155 \\ \mathrm{C} & -4.0349666 & -0.3944938 & 1.5935500 \\ \mathrm{C} & -4.0495207 & 1.5966123 & -0.2398053 \\ \mathrm{H} & 1.1212567 & -2.0682824 & -0.1535882 \\ \mathrm{H} & 3.5990741 & -2.1262173 & -0.1266731 \\ \mathrm{C} & 5.2616134 & 0.0533981 & 0.0643174 \\ \mathrm{H} & 3.5274047 & 2.1672228 & 0.1134651 \\ \mathrm{H} & 1.0546095 & 2.0313923 & 0.0760150 \\ \mathrm{H} & -3.7655111 & -1.4207241 & 1.8294041 \\ \mathrm{H} & -5.1160983 & -0.2647729 & 1.5576419 \\ \mathrm{H} & -3.5675468 & 0.2877975 & 2.3012205 \\ \mathrm{H} & -3.7997555 & 1.9146159 & -1.2486562 \\ \mathrm{H} & -5.1279434 & 1.5444828 & -0.0943110 \\ \mathrm{H} & -3.5733381 & 2.2511883 & 0.4880771 \\ \mathrm{~F} & 5.7657170 & 1.1133187 & -0.5770705 \\ \mathrm{~F} & 5.8117326 & -1.0394823 & -0.4717073 \\ \mathrm{~F} & 5.6897089 & 0.1264720 & 1.3354299\end{array}$

29

COUMPOUND 5: Energy $=-2925.076132$

$\begin{array}{lrrr}\mathrm{Ru} & 0.8589417 & -0.0413998 & 0.0556088 \\ \mathrm{Cl} & 0.7589054 & -1.4428762 & 1.9338027 \\ \mathrm{Cl} & 0.8088652 & -1.9320763 & -1.3466554 \\ \mathrm{Cl} & 0.8758097 & 1.3699257 & -1.8364682 \\ \mathrm{Cl} & 0.8053330 & 1.8293520 & 1.4847559 \\ \mathrm{~S} & 3.1513350 & -0.0348949 & 0.0555373 \\ \mathrm{O} & 3.8694680 & -0.7912020 & 1.0974811 \\ \mathrm{~N} & -1.2524739 & -0.0226383 & 0.0254157 \\ \mathrm{C} & -1.9518525 & -1.1534668 & 0.1706063 \\ \mathrm{C} & -3.3312237 & -1.1779523 & 0.1339647 \\ \mathrm{C} & -4.0246043 & 0.0105697 & -0.0452434 \\ \mathrm{C} & -3.2952646 & 1.1817837 & -0.1928919 \\ \mathrm{C} & -1.9146204 & 1.1223224 & -0.1556516 \\ \mathrm{C} & 3.7955129 & -0.5623137 & -1.5153551 \\ \mathrm{C} & 3.7853195 & 1.6258436 & 0.0779990 \\ \mathrm{H} & -1.3745025 & -2.0537306 & 0.3166229 \\ \mathrm{H} & -3.8620392 & -2.1128990 & 0.2473585 \\ \mathrm{C} & -5.5166774 & -0.0378402 & -0.0955966 \\ \mathrm{H} & -3.7592972 & 2.1451554 & -0.3643855 \\ \mathrm{H} & -1.3118894 & 2.0097434 & -0.2767435 \\ \mathrm{H} & 3.5361042 & -1.6112523 & -1.6326176 \\ \mathrm{H} & 4.8752768 & -0.4200097 & -1.4871092 \\ \mathrm{H} & 3.3286377 & 0.0289832 & -2.3010370 \\ \mathrm{H} & 3.5180617 & 2.0582258 & 1.0386841\end{array}$




$\begin{array}{rrrr}\mathrm{H} & 4.8660963 & 1.5618900 & -0.0432120 \\ \mathrm{H} & 3.3192364 & 2.1882107 & -0.7290696 \\ \mathrm{O} & -6.1773401 & 1.1015283 & 0.0966002 \\ \mathrm{O} & -6.1244109 & -1.0559535 & -0.3046716 \\ \mathrm{H} & -5.5902842 & 1.8300666 & 0.3287358\end{array}$

36

COMPOUND 6: Energy $=-2967.480864$

$\begin{array}{lrrr}\mathrm{Ru} & 1.4916132 & 0.0570217 & -0.0308735 \\ \mathrm{Cl} & 1.4075262 & 2.1033447 & -1.1761741 \\ \mathrm{Cl} & 1.4364571 & 1.2124831 & 2.0214142 \\ \mathrm{Cl} & 1.4943692 & -2.0070941 & 1.1173727 \\ \mathrm{Cl} & 1.4482560 & -1.0669715 & -2.1013591 \\ \mathrm{~S} & 3.7830591 & 0.0446036 & -0.0121449 \\ \mathrm{O} & 4.5143727 & 1.1735173 & -0.6178380 \\ \mathrm{~N} & -0.6212023 & 0.0349179 & -0.0252297 \\ \mathrm{C} & -1.3257637 & 1.1246749 & 0.2958396 \\ \mathrm{C} & -2.7048602 & 1.1393554 & 0.3204071 \\ \mathrm{C} & -3.4218720 & -0.0131555 & 0.0030451 \\ \mathrm{C} & -2.6724970 & -1.1402487 & -0.3303716 \\ \mathrm{C} & -1.2946209 & -1.0778590 & -0.3337324 \\ \mathrm{C} & 4.4118934 & -0.1584635 & 1.6390129 \\ \mathrm{C} & 4.4224280 & -1.4467497 & -0.7397535 \\ \mathrm{H} & -0.7515033 & 2.0042840 & 0.5453810 \\ \mathrm{H} & -3.2098007 & 2.0509291 & 0.6110959 \\ \mathrm{H} & -0.6965358 & -1.9379380 & -0.5959487 \\ \mathrm{H} & 4.1445243 & 0.7360365 & 2.1955682 \\ \mathrm{H} & 5.4927502 & -0.2726148 & 1.5635260 \\ \mathrm{H} & 3.9414812 & -1.0320785 & 2.0865512 \\ \mathrm{H} & 4.1687515 & -1.4228393 & -1.7963309 \\ \mathrm{H} & 5.5013600 & -1.4460019 & -0.5888003 \\ \mathrm{H} & 3.9463017 & -2.3001392 & -0.2600947 \\ \mathrm{H} & -5.0303529 & -2.0969531 & 0.6322405 \\ \mathrm{C} & -5.5825495 & -1.2060290 & 0.3583712 \\ \mathrm{C} & -6.9680601 & -1.2295190 & 0.3777017 \\ \mathrm{H} & -7.4852149 & -2.1408280 & 0.6531603 \\ \mathrm{C} & -7.6903210 & -0.0891188 & 0.0520522 \\ \mathrm{H} & -8.7735485 & -0.1084316 & 0.0646843 \\ \mathrm{C} & -7.0168839 & 1.0761862 & -0.2896932 \\ \mathrm{H} & -7.5724673 & 1.9683613 & -0.5526470 \\ \mathrm{C} & -5.6313212 & 1.1022227 & -0.3023679 \\ \mathrm{H} & -5.1177400 & 2.0121892 & -0.5884799 \\ \mathrm{C} & -4.8946247 & -0.0391924 & 0.0197671 \\ \mathrm{H} & -3.1515179 & -2.0687860 & -0.6109948\end{array}$

39

COMPOUND 7: Energy $=-3006.764021$

$\begin{array}{llrr}\mathrm{Ru} & 1.5038635 & 0.0555845 & -0.0291363 \\ \mathrm{Cl} & 1.4138758 & 2.0953438 & -1.1861381 \\ \mathrm{Cl} & 1.4521400 & 1.2217786 & 2.0171353 \\ \mathrm{Cl} & 1.5144027 & -2.0033504 & 1.1285756 \\ \mathrm{Cl} & 1.4563662 & -1.0796754 & -2.0935526\end{array}$

S31 


$\begin{array}{rrrr}\mathrm{S} & 3.7955474 & 0.0485067 & -0.0150847 \\ \mathrm{O} & 4.5227874 & 1.1806971 & -0.6196687 \\ \mathrm{~N} & -0.6084811 & 0.0300909 & -0.0182353 \\ \mathrm{C} & -1.3143292 & 1.1222659 & 0.2922851 \\ \mathrm{C} & -2.6931459 & 1.1363538 & 0.3165170 \\ \mathrm{C} & -3.4109728 & -0.0194479 & 0.0106267 \\ \mathrm{C} & -2.6594184 & -1.1493251 & -0.3106165 \\ \mathrm{C} & -1.2817860 & -1.0861028 & -0.3148986 \\ \mathrm{C} & 4.4285572 & -0.1560006 & 1.6343887 \\ \mathrm{C} & 4.4375696 & -1.4395576 & -0.7471690 \\ \mathrm{H} & -0.7408828 & 2.0047705 & 0.5333657 \\ \mathrm{H} & -3.1980160 & 2.0508119 & 0.5981084 \\ \mathrm{H} & -0.6834070 & -1.9486423 & -0.5683169 \\ \mathrm{H} & 4.1580413 & 0.7358387 & 2.1937003 \\ \mathrm{H} & 5.5097848 & -0.2647757 & 1.5563124 \\ \mathrm{H} & 3.9632324 & -1.0329734 & 2.0806198 \\ \mathrm{H} & 4.1823912 & -1.4136521 & -1.8033425 \\ \mathrm{H} & 5.5167072 & -1.4367656 & -0.5977133 \\ \mathrm{H} & 3.9640468 & -2.2953012 & -0.2691146 \\ \mathrm{H} & -5.0293375 & -2.1095862 & 0.6201926 \\ \mathrm{C} & -5.5764554 & -1.2133585 & 0.3527914 \\ \mathrm{C} & -6.9590433 & -1.2350646 & 0.3708585 \\ \mathrm{H} & -7.4724603 & -2.1519106 & 0.6398863 \\ \mathrm{C} & -7.7039507 & -0.0982631 & 0.0559915 \\ \mathrm{C} & -9.2007216 & -0.1339307 & 0.0730180 \\ \mathrm{C} & -7.0098571 & 1.0627748 & -0.2729385 \\ \mathrm{H} & -7.5628875 & 1.9596282 & -0.5295938 \\ \mathrm{C} & -5.6243370 & 1.0928405 & -0.2844223 \\ \mathrm{H} & -5.1174288 & 2.0091991 & -0.5626193 \\ \mathrm{C} & -4.8820007 & -0.0458224 & 0.0262108 \\ \mathrm{H} & -3.1371171 & -2.0813259 & -0.5818124 \\ \mathrm{H} & -9.6235578 & 0.8371757 & -0.1839677 \\ \mathrm{H} & -9.5747190 & -0.4183380 & 1.0595682 \\ \mathrm{H} & -9.5832111 & -0.8706787 & -0.6377224\end{array}$

37

COMPOUND 8: Energy $=-3059.656760$

$\begin{array}{lrrr}\mathrm{Ru} & 1.4856610 & 0.0575594 & -0.0286256 \\ \mathrm{Cl} & 1.3960915 & 2.1074714 & -1.1663974 \\ \mathrm{Cl} & 1.4299642 & 1.2052289 & 2.0275924 \\ \mathrm{Cl} & 1.4921966 & -2.0107089 & 1.1113304 \\ \mathrm{Cl} & 1.4405972 & -1.0589218 & -2.1027703 \\ \mathrm{~S} & 3.7771544 & 0.0494375 & -0.0120595 \\ \mathrm{O} & 4.5043075 & 1.1840704 & -0.6114066 \\ \mathrm{~N} & -0.6264823 & 0.0322338 & -0.0210685 \\ \mathrm{C} & -1.3303011 & 1.1208280 & 0.3043022 \\ \mathrm{C} & -2.7097178 & 1.1347090 & 0.3280152 \\ \mathrm{C} & -3.4204691 & -0.0195076 & 0.0080782 \\ \mathrm{C} & -2.6744770 & -1.1464678 & -0.3283765 \\ \mathrm{C} & -1.2964595 & -1.0811698 & -0.3334681 \\ \mathrm{C} & 4.4072838 & -0.1623918 & 1.6373473 \\ \mathrm{C} & 4.4185001 & -1.4358021 & -0.7500068\end{array}$




$\begin{array}{rrrr}\mathrm{H} & -0.7562536 & 1.9996212 & 0.5567517 \\ \mathrm{H} & -3.2169582 & 2.0443455 & 0.6205121 \\ \mathrm{H} & -0.6965142 & -1.9388850 & -0.5987504 \\ \mathrm{H} & 4.1379350 & 0.7278616 & 2.1997121 \\ \mathrm{H} & 5.4883874 & -0.2729779 & 1.5601700 \\ \mathrm{H} & 3.9397909 & -1.0402101 & 2.0796455 \\ \mathrm{H} & 4.1633702 & -1.4057716 & -1.8060678 \\ \mathrm{H} & 5.4976325 & -1.4334273 & -0.6005350 \\ \mathrm{H} & 3.9451438 & -2.2933493 & -0.2750530 \\ \mathrm{H} & -5.0230871 & -2.0981769 & 0.6670747 \\ \mathrm{C} & -5.5757404 & -1.2124853 & 0.3810206 \\ \mathrm{C} & -6.9559245 & -1.2456623 & 0.4017905 \\ \mathrm{H} & -7.4772540 & -2.1496138 & 0.6877865 \\ \mathrm{C} & -7.6770746 & -0.1018860 & 0.0571105 \\ \mathrm{C} & -9.1021364 & -0.1302941 & 0.0727107 \\ \mathrm{C} & -7.0091439 & 1.0690788 & -0.3031464 \\ \mathrm{H} & -7.5716764 & 1.9518154 & -0.5770778 \\ \mathrm{C} & -5.6285398 & 1.0898073 & -0.3138732 \\ \mathrm{H} & -5.1175931 & 1.9962617 & -0.6121949 \\ \mathrm{C} & -4.8930037 & -0.0475608 & 0.0252493 \\ \mathrm{H} & -3.1534562 & -2.0743338 & -0.6109939 \\ \mathrm{~N} & -10.2545094 & -0.1538939 & 0.0849406\end{array}$

26

COMPOUND 1b: Energy $=-2752.268633$

$\begin{array}{lrrr}\mathrm{Rh} & 6.2168512 & 10.1929322 & 3.4653775 \\ \mathrm{Cl} & 4.0909036 & 10.6779563 & 2.6149887 \\ \mathrm{Cl} & 6.0377038 & 12.0197035 & 4.9341794 \\ \mathrm{Cl} & 8.3418446 & 9.6759965 & 4.3469817 \\ \mathrm{Cl} & 6.3235758 & 8.3387336 & 2.0245997 \\ \mathrm{~S} & 7.2405084 & 11.5174087 & 1.8988635 \\ \mathrm{O} & 6.3993229 & 12.2366552 & 0.9314774 \\ \mathrm{~N} & 5.3257365 & 8.9837775 & 4.9049992 \\ \mathrm{C} & 4.2500577 & 9.4025603 & 5.5770357 \\ \mathrm{C} & 3.6420509 & 8.6258822 & 6.5453393 \\ \mathrm{C} & 4.1623709 & 7.3740934 & 6.8297421 \\ \mathrm{C} & 5.2778932 & 6.9456451 & 6.1292779 \\ \mathrm{C} & 5.8317504 & 7.7773570 & 5.1743733 \\ \mathrm{C} & 8.3031963 & 12.7053755 & 2.6789683 \\ \mathrm{C} & 8.4461266 & 10.5875557 & 0.9876274 \\ \mathrm{H} & 3.8829946 & 10.3843660 & 5.3193021 \\ \mathrm{H} & 2.7724550 & 9.0082023 & 7.0629353 \\ \mathrm{H} & 3.7071263 & 6.7440402 & 7.5836691 \\ \mathrm{H} & 5.7239538 & 5.9772924 & 6.3124574 \\ \mathrm{H} & 6.7004813 & 7.4883991 & 4.6022348 \\ \mathrm{H} & 7.6656715 & 13.3932843 & 3.2275418 \\ \mathrm{H} & 8.8506970 & 13.2116790 & 1.8846281 \\ \mathrm{H} & 8.9667583 & 12.1804134 & 3.3631512 \\ \mathrm{H} & 7.9040838 & 9.8639557 & 0.3850046 \\ \mathrm{H} & 8.9936632 & 11.2965305 & 0.3675699 \\ \mathrm{H} & 9.0960026 & 10.0764743 & 1.6953045 \\ & & & \end{array}$




\begin{tabular}{lrrr}
29 & \multicolumn{4}{l}{} \\
COMPOUND 2b: Energy $=-2791.553933$ \\
$\mathrm{Rh}$ & 6.2529708 & 10.2398073 & 3.4170461 \\
$\mathrm{Cl}$ & 4.1257373 & 10.7161337 & 2.5636844 \\
$\mathrm{Cl}$ & 6.0717954 & 12.0787338 & 4.8707740 \\
$\mathrm{Cl}$ & 8.3784743 & 9.7328088 & 4.3026413 \\
$\mathrm{Cl}$ & 6.3621993 & 8.3747370 & 1.9899738 \\
$\mathrm{~S}$ & 7.2756432 & 11.5523533 & 1.8379177 \\
$\mathrm{O}$ & 6.4344139 & 12.2576922 & 0.8600987 \\
$\mathrm{~N}$ & 5.3643685 & 9.0422055 & 4.8643494 \\
$\mathrm{C}$ & 4.2889956 & 9.4606921 & 5.5398612 \\
$\mathrm{C}$ & 3.6841153 & 8.6900757 & 6.5095878 \\
$\mathrm{C}$ & 4.1888906 & 7.4288642 & 6.8165379 \\
$\mathrm{C}$ & 5.3068547 & 7.0132177 & 6.1033902 \\
$\mathrm{C}$ & 5.8631160 & 7.8367561 & 5.1439737 \\
$\mathrm{C}$ & 8.3324695 & 12.7527134 & 2.6072398 \\
$\mathrm{C}$ & 8.4875795 & 10.6178347 & 0.9395607 \\
$\mathrm{H}$ & 3.9193785 & 10.4414242 & 5.2812920 \\
$\mathrm{H}$ & 2.8151402 & 9.0812457 & 7.0241672 \\
$\mathrm{C}$ & 3.5547875 & 6.5722533 & 7.8611242 \\
$\mathrm{H}$ & 5.7546514 & 6.0447818 & 6.2880022 \\
$\mathrm{H}$ & 6.7311974 & 7.5373795 & 4.5758795 \\
$\mathrm{H}$ & 7.6917131 & 13.4426446 & 3.1494599 \\
$\mathrm{H}$ & 8.8782023 & 13.2542616 & 1.8086770 \\
$\mathrm{H}$ & 8.9979459 & 12.2369502 & 3.2965880 \\
$\mathrm{H}$ & 7.9506855 & 9.8804420 & 0.3492096 \\
$\mathrm{H}$ & 9.0287867 & 11.3213858 & 0.3078777 \\
$\mathrm{H}$ & 9.1421855 & 10.1230432 & 1.6544148 \\
$\mathrm{H}$ & 4.0781067 & 5.6234908 & 7.9711561 \\
$\mathrm{H}$ & 2.5125441 & 6.3665863 & 7.6072157 \\
$\mathrm{H}$ & 3.5537319 & 7.0823580 & 8.8269743
\end{tabular}

27

COMPOUND 3b: Energy $=-2844.437780$

$\begin{array}{lrrr}\mathrm{Rh} & 6.2222114 & 10.1947552 & 3.4985759 \\ \mathrm{Cl} & 4.0810917 & 10.6590606 & 2.6740894 \\ \mathrm{Cl} & 6.0559352 & 12.0355037 & 4.9488910 \\ \mathrm{Cl} & 8.3547851 & 9.6913030 & 4.3656945 \\ \mathrm{Cl} & 6.3080063 & 8.3171854 & 2.0898149 \\ \mathrm{~S} & 7.2205979 & 11.4941819 & 1.9017768 \\ \mathrm{O} & 6.3622363 & 12.1872991 & 0.9323780 \\ \mathrm{~N} & 5.3497443 & 9.0003414 & 4.9640782 \\ \mathrm{C} & 4.2789196 & 9.4245703 & 5.6380301 \\ \mathrm{C} & 3.6731542 & 8.6586769 & 6.6138882 \\ \mathrm{C} & 4.2048885 & 7.4056945 & 6.8963060 \\ \mathrm{C} & 5.3217581 & 6.9666876 & 6.1949349 \\ \mathrm{C} & 5.8624917 & 7.7983178 & 5.2351413 \\ \mathrm{C} & 8.2825832 & 12.7019481 & 2.6506433 \\ \mathrm{C} & 8.4203747 & 10.5518972 & 0.9969114 \\ \mathrm{H} & 3.9080856 & 10.4039100 & 5.3772926 \\ \mathrm{H} & 2.8064059 & 9.0357514 & 7.1373648\end{array}$




$\begin{array}{lrrr}\mathrm{H} & 5.7653343 & 6.0000735 & 6.3858960 \\ \mathrm{H} & 6.7282676 & 7.5063349 & 4.6604542 \\ \mathrm{H} & 7.6469160 & 13.3985570 & 3.1903483 \\ \mathrm{H} & 8.8213583 & 13.1946116 & 1.8416942 \\ \mathrm{H} & 8.9549495 & 12.1937447 & 3.3390414 \\ \mathrm{H} & 7.8759252 & 9.8102387 & 0.4193291 \\ \mathrm{H} & 8.9527088 & 11.2509554 & 0.3528900 \\ \mathrm{H} & 9.0847210 & 10.0633798 & 1.7069112 \\ \mathrm{C} & 3.6096660 & 6.5771017 & 7.8965483 \\ \mathrm{~N} & 3.1301666 & 5.9086847 & 8.7014554\end{array}$

29

COMPOUND 4b: Energy $=-3089.143298$

$\begin{array}{lrrr}\text { Rh } & -1.1029186 & -0.0468010 & -0.0505093 \\ \mathrm{Cl} & -1.0217073 & -1.5996989 & -1.7997070 \\ \mathrm{Cl} & -1.0569473 & -1.8100126 & 1.5024908 \\ \mathrm{Cl} & -1.1294963 & 1.5223262 & 1.7080461 \\ \mathrm{Cl} & -1.0685610 & 1.6844575 & -1.6394798 \\ \mathrm{~S} & -3.3905517 & -0.0467229 & -0.0337631 \\ \mathrm{O} & -4.0944621 & -0.9223164 & -0.9801365 \\ \mathrm{~N} & 0.9808711 & -0.0220225 & -0.0325270 \\ \mathrm{C} & 1.6745856 & -1.1599228 & -0.0886175 \\ \mathrm{C} & 3.0570167 & -1.1795365 & -0.0720436 \\ \mathrm{C} & 3.7346701 & 0.0242004 & 0.0037120 \\ \mathrm{C} & 3.0141944 & 1.2053679 & 0.0622844 \\ \mathrm{C} & 1.6346380 & 1.1394345 & 0.0411393 \\ \mathrm{C} & -4.0045441 & -0.3998729 & 1.5929689 \\ \mathrm{C} & -4.0143745 & 1.6006976 & -0.2439025 \\ \mathrm{H} & 1.0961749 & -2.0691421 & -0.1497885 \\ \mathrm{H} & 3.5781162 & -2.1250865 & -0.1237607 \\ \mathrm{C} & 5.2374786 & 0.0580370 & 0.0636020 \\ \mathrm{H} & 3.5017554 & 2.1688755 & 0.1174450 \\ \mathrm{H} & 1.0253198 & 2.0295849 & 0.0812990 \\ \mathrm{H} & -3.7434735 & -1.4305264 & 1.8171527 \\ \mathrm{H} & -5.0842649 & -0.2570141 & 1.5616571 \\ \mathrm{H} & -3.5230955 & 0.2733900 & 2.2994493 \\ \mathrm{H} & -3.7706639 & 1.9094543 & -1.2567516 \\ \mathrm{H} & -5.0915254 & 1.5546523 & -0.0867142 \\ \mathrm{H} & -3.5236335 & 2.2515552 & 0.4772430 \\ \mathrm{~F} & 5.7370096 & 1.1085956 & -0.5960014 \\ \mathrm{~F} & 5.7876037 & -1.0420267 & -0.4562985 \\ \mathrm{~F} & 5.6665893 & 0.1524339 & 1.3323543 \\ & & & \end{array}$

36

COMPOUND 6b: Energy $=-2983.134403$

$\begin{array}{llrr}\text { Rh } & 1.4698812 & 0.0581129 & -0.0268529 \\ \text { Cl } & 1.3984360 & 2.1024168 & -1.1645527 \\ \text { Cl } & 1.4345220 & 1.2087920 & 2.0230404 \\ \text { Cl } & 1.4970413 & -2.0044147 & 1.1163677 \\ \text { Cl } & 1.4366907 & -1.0504307 & -2.0998779 \\ \text { S } & 3.7620912 & 0.0435885 & -0.0148538 \\ \mathrm{O} & 4.4735268 & 1.1745996 & -0.6275655\end{array}$




$\begin{array}{lrrr}\mathrm{N} & -0.6084187 & 0.0350613 & -0.0167893 \\ \mathrm{C} & -1.3081220 & 1.1265744 & 0.3042398 \\ \mathrm{C} & -2.6870108 & 1.1387545 & 0.3264727 \\ \mathrm{C} & -3.4018119 & -0.0148469 & 0.0077785 \\ \mathrm{C} & -2.6522246 & -1.1423741 & -0.3239671 \\ \mathrm{C} & -1.2745547 & -1.0805110 & -0.3260740 \\ \mathrm{C} & 4.3797985 & -0.1527711 & 1.6372323 \\ \mathrm{C} & 4.3801244 & -1.4499339 & -0.7473530 \\ \mathrm{H} & -0.7315140 & 2.0043043 & 0.5538690 \\ \mathrm{H} & -3.1932440 & 2.0497703 & 0.6162738 \\ \mathrm{H} & -0.6724865 & -1.9379412 & -0.5868377 \\ \mathrm{H} & 4.1237992 & 0.7507114 & 2.1839167 \\ \mathrm{H} & 5.4587687 & -0.2831273 & 1.5598838 \\ \mathrm{H} & 3.8945791 & -1.0159773 & 2.0884977 \\ \mathrm{H} & 4.1335192 & -1.4139599 & -1.8049480 \\ \mathrm{H} & 5.4576307 & -1.4613666 & -0.5864619 \\ \mathrm{H} & 3.8871767 & -2.2971494 & -0.2744283 \\ \mathrm{H} & -5.0108226 & -2.0981435 & 0.6370674 \\ \mathrm{C} & -5.5625161 & -1.2078885 & 0.3600695 \\ \mathrm{C} & -6.9480264 & -1.2314420 & 0.3760401 \\ \mathrm{H} & -7.4658401 & -2.1421926 & 0.6519970 \\ \mathrm{C} & -7.6694245 & -0.0917523 & 0.0460079 \\ \mathrm{H} & -8.7526771 & -0.1113113 & 0.0556294 \\ \mathrm{C} & -6.9955626 & 1.0731229 & -0.2963382 \\ \mathrm{H} & -7.5506995 & 1.9645371 & -0.5626723 \\ \mathrm{C} & -5.6100337 & 1.0995864 & -0.3050444 \\ \mathrm{H} & -5.0958533 & 2.0090991 & -0.5914765 \\ \mathrm{C} & -4.8744059 & -0.0413214 & 0.0211033 \\ \mathrm{H} & -3.1304509 & -2.0710597 & -0.6050362\end{array}$

39

COMPOUND 7b: Energy $=-3022.417391$

$\begin{array}{lrrr}\text { Rh } & 1.4818331 & 0.0561035 & -0.0395423 \\ \mathrm{Cl} & 1.4107114 & 2.0526160 & -1.2577936 \\ \mathrm{Cl} & 1.4610092 & 1.2900858 & 1.9609795 \\ \mathrm{Cl} & 1.5020828 & -1.9580828 & 1.1851701 \\ \mathrm{Cl} & 1.4450129 & -1.1346347 & -2.0670627 \\ \mathrm{~S} & 3.7737113 & 0.0340954 & -0.0295610 \\ \mathrm{O} & 4.4865376 & 1.1444603 & -0.6771657 \\ \mathrm{~N} & -0.5955587 & 0.0385957 & -0.0236884 \\ \mathrm{C} & -1.2946709 & 1.1326691 & 0.2902999 \\ \mathrm{C} & -2.6731768 & 1.1442658 & 0.3226694 \\ \mathrm{C} & -3.3921819 & -0.0107723 & 0.0147784 \\ \mathrm{C} & -2.6415802 & -1.1393158 & -0.3145953 \\ \mathrm{C} & -1.2643637 & -1.0789572 & -0.3199898 \\ \mathrm{C} & 4.3945498 & -0.1156559 & 1.6265856 \\ \mathrm{C} & 4.3888150 & -1.4809585 & -0.7187666 \\ \mathrm{H} & -0.7173515 & 2.0127052 & 0.5299045 \\ \mathrm{H} & -3.1754671 & 2.0575552 & 0.6117391 \\ \mathrm{H} & -0.6643252 & -1.9390392 & -0.5763924 \\ \mathrm{H} & 4.1391432 & 0.8020271 & 2.1492503 \\ \mathrm{H} & 5.4733681 & -0.2478448 & 1.5506912\end{array}$




$\begin{array}{lrrr}\mathrm{H} & 3.9108266 & -0.9666324 & 2.1019914 \\ \mathrm{H} & 4.1460248 & -1.4720352 & -1.7777735 \\ \mathrm{H} & 5.4657702 & -1.4911580 & -0.5541955 \\ \mathrm{H} & 3.8916872 & -2.3141203 & -0.2258432 \\ \mathrm{H} & -5.0051672 & -2.1301191 & 0.5338312 \\ \mathrm{C} & -5.5539765 & -1.2239281 & 0.3072575 \\ \mathrm{C} & -6.9362376 & -1.2521365 & 0.3225755 \\ \mathrm{H} & -7.4459212 & -2.1829131 & 0.5470465 \\ \mathrm{C} & -7.6854252 & -0.1048333 & 0.0606898 \\ \mathrm{C} & -9.1819073 & -0.1492855 & 0.0656505 \\ \mathrm{C} & -6.9955035 & 1.0737089 & -0.2099492 \\ \mathrm{H} & -7.5516700 & 1.9802231 & -0.4218199 \\ \mathrm{C} & -5.6102519 & 1.1093184 & -0.2207583 \\ \mathrm{H} & -5.1083964 & 2.0405065 & -0.4547274 \\ \mathrm{C} & -4.8631440 & -0.0402262 & 0.0345939 \\ \mathrm{H} & -3.1186357 & -2.0698403 & -0.5906238 \\ \mathrm{H} & -9.6091463 & 0.8432147 & -0.0767745 \\ \mathrm{H} & -9.5597145 & -0.5534094 & 1.0075273 \\ \mathrm{H} & -9.5555207 & -0.7964400 & -0.7321194\end{array}$

37

COMPOUND 8b: Energy $=-3075.314791$

$\begin{array}{lrrr}\mathrm{Rh} & 1.4642933 & 0.0566122 & -0.0252104 \\ \mathrm{Cl} & 1.3890032 & 2.1024450 & -1.1624881 \\ \mathrm{Cl} & 1.4294277 & 1.2042581 & 2.0260023 \\ \mathrm{Cl} & 1.4909167 & -2.0054444 & 1.1156954 \\ \mathrm{Cl} & 1.4316669 & -1.0481262 & -2.0996367 \\ \mathrm{~S} & 3.7556296 & 0.0452507 & -0.0133052 \\ \mathrm{O} & 4.4577371 & 1.1842716 & -0.6234077 \\ \mathrm{~N} & -0.6156663 & 0.0319958 & -0.0148364 \\ \mathrm{C} & -1.3149954 & 1.1220339 & 0.3115839 \\ \mathrm{C} & -2.6940703 & 1.1335496 & 0.3339364 \\ \mathrm{C} & -3.4031097 & -0.0212348 & 0.0119660 \\ \mathrm{C} & -2.6570475 & -1.1484133 & -0.3238147 \\ \mathrm{C} & -1.2793594 & -1.0837842 & -0.3283093 \\ \mathrm{C} & 4.3787376 & -0.1542593 & 1.6361209 \\ \mathrm{C} & 4.3804618 & -1.4405546 & -0.7549404 \\ \mathrm{H} & -0.7404161 & 1.9998974 & 0.5647720 \\ \mathrm{H} & -3.2014032 & 2.0428228 & 0.6268543 \\ \mathrm{H} & -0.6775336 & -1.9400679 & -0.5929057 \\ \mathrm{H} & 4.1173442 & 0.7435665 & 2.1895078 \\ \mathrm{H} & 5.4585225 & -0.2745286 & 1.5540100 \\ \mathrm{H} & 3.9043715 & -1.0252728 & 2.0837701 \\ \mathrm{H} & 4.1278819 & -1.4043487 & -1.8110985 \\ \mathrm{H} & 5.4589186 & -1.4425950 & -0.6002106 \\ \mathrm{H} & 3.8995736 & -2.2938731 & -0.2806140 \\ \mathrm{H} & -5.0067260 & -2.1026171 & 0.6611406 \\ \mathrm{C} & -5.5584114 & -1.2156240 & 0.3773419 \\ \mathrm{C} & -6.9386826 & -1.2483614 & 0.3964529 \\ \mathrm{H} & -7.4609019 & -2.1526937 & 0.6796144 \\ \mathrm{C} & -7.6584364 & -0.1032493 & 0.0534584 \\ \mathrm{C} & -9.0835761 & -0.1309346 & 0.0673350\end{array}$




$\begin{array}{lrrr}\mathrm{C} & -6.9902859 & 1.0686458 & -0.3032082 \\ \mathrm{H} & -7.5524152 & 1.9521207 & -0.5755212 \\ \mathrm{C} & -5.6096336 & 1.0890737 & -0.3118162 \\ \mathrm{H} & -5.0982933 & 1.9961134 & -0.6076768 \\ \mathrm{C} & -4.8756356 & -0.0495481 & 0.0259587 \\ \mathrm{H} & -3.1346966 & -2.0769400 & -0.6059274 \\ \mathrm{~N} & -10.2359601 & -0.1533562 & 0.0786766\end{array}$

30

COMPOUND 2' $-\mathrm{Na}^{+}$: Energy $=-2938.048982$

$\begin{array}{lrrr}\mathrm{Ru} & 1.1930620 & -4.3942992 & 1.8498455 \\ \mathrm{Cl} & 1.7611428 & -3.2030805 & 3.8339531 \\ \mathrm{Cl} & -1.0492800 & -3.8010956 & 2.1040880 \\ \mathrm{Cl} & 0.6655964 & -5.6099833 & -0.0701878 \\ \mathrm{Cl} & 3.4633967 & -5.0847873 & 1.6486070 \\ \mathrm{~S} & 1.6822495 & -2.4768376 & 0.6857413 \\ \mathrm{O} & 3.0186468 & -1.8873537 & 0.9682677 \\ \mathrm{~N} & 0.8174758 & -6.1202294 & 3.0004155 \\ \mathrm{C} & 1.2807776 & -7.3179589 & 2.6270075 \\ \mathrm{C} & 1.0660675 & -8.4610478 & 3.3686699 \\ \mathrm{C} & 0.3414597 & -8.3984732 & 4.5547792 \\ \mathrm{C} & -0.1308673 & -7.1454235 & 4.9311516 \\ \mathrm{C} & 0.1219717 & -6.0438209 & 4.1399060 \\ \mathrm{C} & 0.0657449 & -9.6177643 & 5.3691840 \\ \mathrm{C} & 0.5066226 & -1.1795958 & 0.9641438 \\ \mathrm{C} & 1.5707370 & -2.6437036 & -1.0744703 \\ \mathrm{H} & 1.8392076 & -7.3463379 & 1.7033693 \\ \mathrm{H} & 1.4714934 & -9.4005970 & 3.0142625 \\ \mathrm{H} & -0.6971045 & -7.0164903 & 5.8451161 \\ \mathrm{H} & -0.2373726 & -5.0634491 & 4.4150902 \\ \mathrm{H} & 0.8727960 & -10.3450052 & 5.2789177 \\ \mathrm{H} & -0.0748657 & -9.3691100 & 6.4209673 \\ \mathrm{H} & -0.8511131 & -10.1007153 & 5.0191915 \\ \mathrm{H} & 0.5055309 & -0.9800578 & 2.0340197 \\ \mathrm{H} & -0.4790823 & -1.5103971 & 0.6461851 \\ \mathrm{H} & 0.8477283 & -0.3118114 & 0.4006670 \\ \mathrm{H} & 2.2947597 & -3.3986428 & -1.3731250 \\ \mathrm{H} & 0.5667389 & -2.9690332 & -1.3386385 \\ \mathrm{H} & 1.8096211 & -1.6731498 & -1.5080214 \\ \mathrm{Na} & 4.2281040 & -2.7098035 & 2.7227387\end{array}$

44

COMPOUND 2'-BH ${ }^{+}(\mathrm{Cl}):-3063.735306021$

$\begin{array}{lrrr}\mathrm{Ru} & 0.2502280 & 1.8863870 & 5.6874230 \\ \mathrm{Cl} & -0.0812500 & 4.0294710 & 4.7703360 \\ \mathrm{Cl} & -1.4224620 & 2.3652300 & 7.2802950 \\ \mathrm{Cl} & 0.5315500 & -0.2709540 & 6.5449570 \\ \mathrm{Cl} & 1.8672220 & 1.4892250 & 4.0101000 \\ \mathrm{~S} & 1.8467340 & 2.5628130 & 7.1908640 \\ \mathrm{O} & 3.2488120 & 2.2438590 & 6.8763090 \\ \mathrm{~N} & -1.2114580 & 1.1350020 & 4.3694440 \\ \mathrm{C} & -1.0192130 & -0.0193140 & 3.7172020\end{array}$




$\begin{array}{lrrr}\mathrm{C} & -1.9834600 & -0.5719520 & 2.8891930 \\ \mathrm{C} & -3.1884150 & 0.0525640 & 2.7123940 \\ \mathrm{C} & -3.3778000 & 1.2366080 & 3.3915970 \\ \mathrm{C} & -2.3889340 & 1.7618540 & 4.1916130 \\ \mathrm{C} & -4.2525940 & -0.5362290 & 1.8298710 \\ \mathrm{C} & 1.5601400 & 1.9341010 & 8.8296450 \\ \mathrm{C} & 1.7925900 & 4.2849640 & 7.5331170 \\ \mathrm{H} & -0.0645965 & -0.4986986 & 3.8706616 \\ \mathrm{H} & -1.7594881 & -1.5066232 & 2.3889649 \\ \mathrm{H} & -4.3084523 & 1.7830705 & 3.2958322 \\ \mathrm{H} & -2.5181324 & 2.6922462 & 4.7239011 \\ \mathrm{H} & -4.5596080 & -1.5146531 & 2.2055323 \\ \mathrm{H} & -5.1295047 & 0.1076616 & 1.7815997 \\ \mathrm{H} & -3.8710118 & -0.6840125 & 0.8176637 \\ \mathrm{H} & 1.6309114 & 0.8506816 & 8.7749002 \\ \mathrm{H} & 2.3354191 & 2.3537609 & 9.4693462 \\ \mathrm{H} & 0.5631411 & 2.2269510 & 9.1518279 \\ \mathrm{H} & 2.0183751 & 4.8110520 & 6.6082500 \\ \mathrm{H} & 2.5338005 & 4.4983112 & 8.3024092 \\ \mathrm{H} & 0.7868768 & 4.5415952 & 7.8634230 \\ \mathrm{~N} & 4.1123130 & -0.2356750 & 6.0259050 \\ \mathrm{C} & 5.3652840 & -0.1917980 & 6.4384360 \\ \mathrm{C} & 6.2141240 & -1.2091410 & 6.1143040 \\ \mathrm{C} & 5.7656430 & -2.2840200 & 5.3688010 \\ \mathrm{C} & 4.4415900 & -2.2794260 & 4.9768960 \\ \mathrm{C} & 3.6400470 & -1.2471270 & 5.3157610 \\ \mathrm{C} & 6.6702060 & -3.4315800 & 5.0019430 \\ \mathrm{H} & 3.5218672 & 0.5874539 & 6.2354452 \\ \mathrm{H} & 5.6609364 & 0.6772132 & 7.0085091 \\ \mathrm{H} & 7.2423785 & -1.1592588 & 6.4470533 \\ \mathrm{H} & 4.0362284 & -3.0928821 & 4.3898480 \\ \mathrm{H} & 2.5994652 & -1.1696203 & 5.0354372 \\ \mathrm{H} & 7.7181208 & -3.1408408 & 5.0515001 \\ \mathrm{H} & 6.4384913 & -3.8001494 & 4.0030129 \\ \mathrm{H} & 6.5116279 & -4.2548177 & 5.7036916\end{array}$

\begin{tabular}{llcl}
44 & \multicolumn{4}{l}{} \\
COMPOUND & $2^{\prime}-\mathrm{BH}^{+}$(arom) : Energy & -3063.724027 \\
$\mathrm{~N}$ & 4.299665 & 4.593975 & 0.772023 \\
$\mathrm{C}$ & 3.406089 & 3.994773 & 1.549939 \\
$\mathrm{C}$ & 2.446648 & 4.705027 & 2.179050 \\
$\mathrm{C}$ & 2.388920 & 6.084128 & 2.027297 \\
$\mathrm{C}$ & 3.350943 & 6.667581 & 1.211074 \\
$\mathrm{C}$ & 4.288794 & 5.908904 & 0.598170 \\
$\mathrm{C}$ & 1.334639 & 6.889451 & 2.699134 \\
$\mathrm{H}$ & 4.956616 & 4.072874 & 0.327078 \\
$\mathrm{H}$ & 3.450404 & 3.062864 & 1.657492 \\
$\mathrm{H}$ & 1.828597 & 4.269899 & 2.724180 \\
$\mathrm{H}$ & 3.361274 & 7.596009 & 1.084368 \\
$\mathrm{H}$ & 4.926136 & 6.305955 & 0.053040 \\
$\mathrm{H}$ & 1.339200 & 6.685268 & 3.631749 \\
$\mathrm{H}$ & 1.509907 & 7.821936 & 2.584214
\end{tabular}




$\begin{array}{lrrr}\mathrm{H} & 0.478655 & 6.680856 & 2.336696 \\ \mathrm{Ru} & 5.960185 & 10.274502 & 1.761347 \\ \mathrm{Cl} & 5.794814 & 11.624668 & 3.670203 \\ \mathrm{Cl} & 3.634244 & 10.523922 & 1.426917 \\ \mathrm{Cl} & 6.089882 & 8.910672 & -0.143649 \\ \mathrm{Cl} & 8.250831 & 9.992667 & 2.219124 \\ \mathrm{~S} & 6.422372 & 12.004736 & 0.339749 \\ \mathrm{O} & 7.790947 & 12.073778 & -0.212896 \\ \mathrm{~N} & 5.601136 & 8.574441 & 2.974646 \\ \mathrm{C} & 6.306634 & 7.449116 & 2.793427 \\ \mathrm{C} & 6.084919 & 6.301508 & 3.531563 \\ \mathrm{C} & 5.107370 & 6.272053 & 4.499539 \\ \mathrm{C} & 4.401789 & 7.439950 & 4.692544 \\ \mathrm{C} & 4.659240 & 8.549507 & 3.927888 \\ \mathrm{C} & 4.808378 & 5.037175 & 5.293661 \\ \mathrm{C} & 6.074039 & 13.596157 & 1.025435 \\ \mathrm{C} & 5.312130 & 12.028334 & -1.049008 \\ \mathrm{H} & 6.982286 & 7.453140 & 2.139270 \\ \mathrm{H} & 6.604043 & 5.535498 & 3.368024 \\ \mathrm{H} & 3.734225 & 7.470666 & 5.348174 \\ \mathrm{H} & 4.172819 & 9.338325 & 4.061961 \\ \mathrm{H} & 5.621826 & 4.563596 & 5.454254 \\ \mathrm{H} & 4.421146 & 5.297160 & 6.126091 \\ \mathrm{H} & 4.197062 & 4.492692 & 4.795677 \\ \mathrm{H} & 6.608693 & 13.727486 & 1.798931 \\ \mathrm{H} & 5.155166 & 13.646136 & 1.262641 \\ \mathrm{H} & 6.272944 & 14.269755 & 0.386011 \\ \mathrm{H} & 5.385603 & 11.215516 & -1.535206 \\ \mathrm{H} & 4.420964 & 12.134465 & -0.739610 \\ \mathrm{H} & 5.538742 & 12.758084 & -1.616239\end{array}$

